\title{
INDEX TO VOLUME 126
}

This index provides coverage for both the Initial Reports and Scientific Results portions of Volume 126 of the Proceedings of the Ocean Drilling Program. References to page numbers in the Initial Reports are preceded by "A" with a colon (A:), and those in the Scientific Results (this book), by "B" with a colon (B:).

The index was prepared by Wm. J. Richardson Associates, Inc., under subcontract to the Ocean Drilling Program. The index contains two hierarchies of entries: (1) a main entry, defined as a keyword or concept followed by a reference to the page on which that word or concept appears, and (2) a subentry, defined as a further elaboration on the main entry followed by a page reference.

The index is presented in two parts: (1) a Subject Index and (2) a Taxonomic Index. Both parts cover text figures and tables but not core-description forms ("barrel sheets") or core photographs. Also excluded are bibliographic references, names of individuals, and routine front and back matter.

The Subject Index follows a standard format. Geographic, geologic, and other terms are referenced only if they are subjects of discussion. This index also includes taxonomic entries above the generic level, as well as broad fossil groups such as foraminifers and radiolarians. The notation "ff" following a page listing indicates that reference to a topic continues beyond the last page given but is not sequential. This would be the case where one or more figures or tables follow a principal topic of discussion that makes up a major section. A site chapter in the Initial Reports is considered the principal reference for that site and is indicated on the first line of the site's listing in the index. Such a reference to Site 787, for example, is given as "Site 787, A:63-96."

The Taxonomic Index is an index relating to significant findings and/or substantive discussions, not of species names per se. This index covers three varieties of information: (1) individual genera and species that have been erected or emended formally, (2) biostratigraphic zones, and (3) fossils depicted in illustrations. A taxonomic entry consisting of both genus and species is listed alphabetically by genus and also by species. Biostratigraphic zones are listed alphabetically by genus; zones with letter prefixes are listed under "zones."

For further information, including available electronic formats, contact the Chief Production Editor, Ocean Drilling Program, 1000 Discovery Drive, College Station, Texas 77845-9547, U.S.A. 
accretion, Izu-Bonin-Mariana Arc, B:632 acidic volcanics, in hemipelagic sediments, B:498 acoustic basement, seismic reflection profiling, A: 132

acoustic index, B:547-549

alanine, Sumisu Rift, B:537

albite, B:522

alkaline earth concentration, Sumisu Rift, B:394 alkalinity

carbonate precipitation and, A:378

Site 787, A:88

Sites 790/791, A:187-188

alteration

geochemical effects, A:269, 372, 378

posteruptive, A:262

"snowflake," A:334-335

volcanic rock, B:115

alteration, diagenetic, volcaniclastic sandstones, B:125-132

alteration, hydrothermal

basement, B:392

geochemical effects, A:266

Izu-Bonin forearc, B:101-107

secondary mineralogy, B:105

Sumisu Rift, B:190-191

volcaniclastic rock, B:419, 436-439, 442

alteration, low-temperature, basement, A:265

aluminosilicates, as calcium sink, B:523

aluminum

basement, B:433-434, 437

logging data, A:206; B:655-656

aluminum oxide

basement, B:390

core vs. log measurements, B:593, 597

magnesium oxide correlation, A:370

in pelagic/hemipelagic sediments, B:500

in volcaniclastic sand/sandstones, B:470

aluminum oxide/magnesium oxide ratio, basement, B:409, 423

aluminum oxide/zirconium ratio, basement, B:416

alunite, X-ray diffraction, A:150

Alutom Formation (Guam), B:632

amino acids. See also dissolved combined amino acids (DCAA); dissolved free amino acids (DFAA)

aromatic and sulfur-containing, B:532, 536

biogeochemistry, B:537-538

nonprotein, B:537-538

source material, B:536-537

Sumisu Rift, B:532-540

amino butyric acid, Sumisu Rift, B:537-538

ammonia

Site 787, A:88

Site 793, A:378

ammonium

Site 790, A:188

Site 791, A:188, 194

Site 792, A: 270

amphibole, Site 792, B:678

analcime veins, Ohmachi Seamount, B:189

analcite. See cement, analcite

andesite

aluminum oxide-magnesium oxide correlation, A:370

aphyric to sparsely phyric, A:367; B:432, 438

basement, B:444

boninitic affinity, B:442

chromite-olivine, B:438

clinopyroxene-orthopyroxene, B:233

geochemistry, B:419, 421, 426, 429, 432, 442 glass inclusions, B:171-175

lithologic types, A:367, 369, 371

magnesium-chromium-nickel relationship,

$$
\text { A:370 }
$$

olivine- and chromium-spinel-bearing, A:367; B:432

phenocryst minerals, B:419, 421

plagioclase composition, B:433

rhyolite transition, oceanic arcs, B:395

Site 788, A:120

Site 792, B:677

trace elements, A:370-371; B:419

types i-iv, B:432, 434, 442

in volcanic breccias, A:361-362

andesite, porphyritic

calc-alkaline affinity, B:442

clinopyroxene-rich, A:367

Izu-Bonin forearc, B:171-172, 442

petrology, A:262-266, 361

plagioclase-rich, A:367; B:432

pyroxene-rich, B:432

xenolith, A:267

andesite, two-pyroxene

Izu-Benin forearc, B:431

porphyritic, B:450-451

Site 792, B:419, 430

andesite class

geochemistry, B:386-387

Site 787, A:69

in volcaniclastic breccia, B:433

andesite intrusions, age, B:632

anorthite, in plagioclase, B:174, 523

Aoga Shima Canyon

bathymetry, A:66

multichannel seismic survey, A:54-55

sandy gravel, B:640

unconsolidated volcaniclastics, A:75, 77

Aoga Shima Rift, arc margin uplift, B:646

Aoga Shima Islands

basalt, B:63

volcaniclastics, B:34

arc rifting

initiation, B:648

Izu-Bonin Arc, B:640, 642-644, 646

Izu-Bonin-Mariana Arc, B:627, 629, 632-636

location control, B:564, 570-571, 647-648

Mariana region, B:647

volcanism and, B:647-648

vs. spreading, B:383

arc volcanism

backarc, B:639, 647-648

cyclic model, B:648

forearc, B:639

frontal arc, B:639

Izu-Bonin Arc, B:584, 646

Izu-Bonin-Mariana Arc, B:632, 638-639

post-rift-inception, B:387-388

pre-rift, B:386-388

on proto-remnant arc, B:395

pyroclastic, B:639

arginine, Sumisu Rift, B:538

ash, black sandy

in nannofossil-rich claystone, A:72

Site 787, A:74

ash, pumiceous, Site 793, A:328

ash, sandy, X-ray diffraction, A:148

ash, vitric silty, Site 793, A:331

ash, volcanic

Site 790, A:142-144

transport mechanisms, A:158 ash, volcaniclastic, depositional process, B:228

ash, white, Site 790, A:150

ash beds

in nannofossil-rich clay, A:154

Site 793, A:331

ash layers

accumulation rate, $\mathrm{B}: 27,33$

age, B:15

chemistry, B:40

colors, B:27

deposition, B:225

grain-size distribution, $\mathrm{B}: 27,34$

Izu-Bonin Arc, B:23-35, 43-45

oxide stratigraphy, B:512

sediment source, B:515-516

Site 791, A:147

Site 792, A:232-236, 238

Sumisu Rift, B:10

thickness, B:27

ash tuff

petrology, A:174

X-ray diffraction, A:186

Aso Volcano

glass chemistry, B:34-35

marine tephra, B:42

aspartic acid, decomposition, B:538

augite, in volcaniclastic sand/sandstone, B:160

backarc basin basalt (BABB)

geochemistry, B:389, 642

magma composition, B:383

in rift basins, B:555

source, B:393

backarc basins

hydrothermal circulation, B:555, 557

Izu-Bonin Arc, B:557

sedimentation models, B:3

vein structures, B:205

backarc rifting, geochemical effects, B:482

backarc spreading, B:636, 638-639

arc volcanism and, B:47, 62, 647-648

manganese deposits, B:113

Mariana vs. Izu-Bonin systems, A:5; B:647

Philippine Sea, B:47

volcanism and, A:8-9; B:483

backarc spreading centers, manganese deposits, B:113

barium

in basement basalts, A:184

in manganese minerals, B:115

barium/strontium ratio

Izu-Ogasawara Arc, B:465

tephras, B:462

barium/zirconium ratio, Site 791, A:190

basalt

arc vs, rift, B:395-396

petrology, A:176-177, 183

rift basement, A:128-129

basalt, basement

geochemistry, A:184; B:397-398, 407-417

island-arc origin, B:583

sulfur isotopes, B:450, 452

tholeiitic affinity, A:370

vesicle size distribution, B:403

basalt, glomeroporphyritic, Site 791, A:176

basalt, olivine-plagioclase, Site 791, A:176-177

basalt clasts

geochemistry, B:386

Site 791, A:148

basalt detritus, source, A:157 
basalt flow, petrology, A:174

basalt pebbles, geochemistry, B:420

basaltic mousse

description, B:371

emplacement mechanisms, B:373, 379

geochemistry, B:371, 391, 401, 404, 451

juvenile glass clasts, B:450

magnetic properties, A:170-171; B:371-377

origin, B:371, 373, 375, 379

petrology, A:174, 179, 181; B:391-392

physical properties, A:200

Site 790, A:415

Site 791, A:415; B:371-372, 400

sulfate-sulfur concentration, B:450

sulfur isotopes, B:452

vesicular components, B:391

$\mathrm{X}$-ray diffraction, A:184

basement. See also acoustic basement; lava, basement

age, A:174; B:388, 677-680

brecciation, A:407

forearc, A:6

geochemistry, A:269-271; B:410-413, 418

hydrothermal alteration, B:392

igneous geochemistry, A:369-371

igneous stratigraphy, A:176, 362-367; B:408, 439-440

Izu-Bonin forearc, A:223-224

lithologic types, A:367, 369

lithostratigraphy, A:180, 366; B76

low-temperature alteration, A:265

magnetic properties, A:263

mineralogy, A:264; B:409

petrology, A:262-263, 362-367

phenocrysts, A:365

seismic reflection profiling, A:6-7

stratigraphic summary, A:265; B:421

subsidence, B:630

vertical motion history, B:299-301

basement, volcanic

geochemistry, B:419, 421, 424-425, 483

igneous stratigraphy, B:405, 419

lithologies, B:634

petrography, $\mathrm{B}: 432$

stable isotopes, B:421-422

basement/sediment contact

fluid circulation, B:619

logging data, A:289

seismic reflection profiling, B:580

sonic velocity, A:284

bed thickness/maximum particle size ratio, IzuBonin forearc, B:97, 99

bedding parallel slip, Izu-Bonin forearc, B:205206

bioclast pebbles, Izu-Benin forearc, B:87

bioclasts, B:141

biomicrite, Site 793, B:231-232, 234

biosparite, Site 793, B:232-233

Biwa II excursion, Site 790, A:169

Blake Event, Site 790, A:169

Bonin Arc, rifting, A:5

Bonin forearc, basement A:6-7

Bonin Islands

boninite, B:497

declination anomalies, B:353

formation, A:9

intraoceanic forearc basement, A:6

outer-arc high, B:467

potassium-argon dating, B:632

uplift-subsidence history, A:8; B:630

volcaniclastic sandstone, B:483

Bonin Ridge, uplift, B:630, 640

boninite formation, A:6

intrusion age, B:632

origin, $\mathrm{B}: 422$

boninite series volcanics (BSV), forearc terrains, B:422

border faults

across transfer zones, B:566

Izu-Bonin-Mariana Arc, B:634

Sumisu Rift, B:568-570, 642

zigzag pattern, B:564

Boso Peninsula

Brunhes/Matuyama reversal, B:346

Shirahama formation, B:49

virtual magnetic path, B:349

breccia, andesite, petrology, A:364-365

breccia, andesitic hyaloclastite

petrology, A:264; B:439

Site 792, B:172

breccia, basalt, Site 791, A:176

breccia, basaltic andesite, mineralogy, B:105, 110

breccia, basement, phenocryst assemblages, B:406

breccia, hyaloclastite

mineralogy, B:105, 111

Site 792, B:419

breccia, monolithic, petrology, A:363-364

breccia, volcanic

derivation, $\mathrm{A}: 348$

heterolithic, B:406

logging data, B:657

metamorphism, B:186

petrography, B:439

petrology, A:264

Site 793, A:337-338; B:405-406

breccia, volcaniclastic

andesite clasts, B:442

geochemistry, A:369

mineralogy, B:433

petrography, B:432

brine, calcium chloride, Site 792, B:519

bronzite, in basaltic andesite, $\mathrm{B}: 432$

Brunhes/Matuyama boundary, B:341-351

Site 790, A:170

Site 791, A:163

Brunhes Normal Chronozone, Site 792, A:255

bryozoans, Site 793, B:231

burrows

Izu-Bonin forearc, B:210, 212, 213, 215

Site 791, A:148, 160

Site 793, A:330-331

buserite, Izu-Bonin forearc, B:115

calcite

Izu-Bonin forearc, B:101-102

Site 792, A:240

Site 793, A:338, 340

smectite covering, B:135

calcium

alteration effects, A:269, 378

basement, B:434, 437

concentration gradient, B:520-521

deep maximum, A:269-270

gypsum precipitation and, B:521

logging data, A:206

in manganese minerals, B:115

sinks, B:521-523

Site 787, A:88

Sites 790/791, A:187-188

Site 793, A:372

calcium carbonate, B:489, 495

density and, A:189

grain density, B:553

logging data, B:656, 652-663, 668-669

Site $788, A: 123$
Site 790, A:195

Site 791, A:200

Site 792, A:219

Site 793, A:332

turbidite influence, A:92

calcium chloride, in pelagic/hemipelagic sediments, B:499

calcium/magnesium ratio

basement, B:409, 423

in manganese deposits, $\mathrm{B}: 117$

Site 791, A:188

Site 792, A:269

calcium oxide

core vs. log measurements, B:597

logging data B:657

in pelagic/hemipelagic sediments, B:499

calcium/zirconium ratio, basement, B:416

caldera submarine

Izu-Bonin Arc, B:3, 10

magma composition, B:387

canyon, submarine

erosion, B:576, 640

sediment till, B:582

carbon, inorganic, Site 793, A:374-378

carbon, organic

carbonate correlation, A:186

Site 787, A:85

Site 788, A:122

Sites 790/791, A:186, 191-193

Site 792, A:268, 272-276

Site 793, A:374-375, 378-379

carbon, total

Site 787, A:88

Sites 790/791, A:186

Site 792, A:268

Site 793, A:374-378

carbonate

alkalinity and precipitation of, A:378

density and, A:122-123, 379

depositional environment, A:115

high-to-low transition, A:155

in pelagic/hemipelagic sediments, B:493, 496

Site 788, A:109, 126

Sites 790/791, A:168

Site 792, A:272-276

Site 793, A:327-328, 374-379

carbonate bioclasts

depositional environment, B:233

Site 793, B:231-233

source, B:233

Caroline Plate, subduction, B:647

Cascade region

bimodal volcanism, B:35, 62-63

silica, B:60

celadonite

Site 793, A:342

vs. glauconite, A:114

Celebes Sea Basin, viscous remanent magnetization, B:357

cement, analcite, precipitation temperature, B:132

cement, carbonate

Izu-Bonin forearc, B:128

Site 788, A:111, 116

cement, clay-mineral

in Oligocene sandstones, B:128

Site 788, B:137

cement, clinoptilolite, precipitation temperature, B: 132

cement, phillipsite, Site 788, B:126

cement, zeolite, B:131

cementation

physical property effects of, B:545

Site 793, A:334 
cerium, negative anomaly, B:396 cerium/lead ratio, Izu Arc, B:387

cerium/ytterbium ratio

Site 792, B:461

tholeiitic layers, B:465

vs. tantalum/ytterbium ratio, B:479, 481

chabazite, B:523-525

chalk, nannofossil

depositional environment, A:246

Site 787, A:69

Site 792, A:235; B:225

Chichijima Island

boninitic-andesitic rock, B:497

foraminifers, B:233

magnetic anomalies, B:359

volcanic highs, B:630

chloride

Miocene/Oligocene boundary gradient, B:519520

Site $787, \mathrm{~A}: 85$

Site 793, A:372

chlorine, logging data, A:203, 205, 211-215

chlorite

chemical composition, B:187, 189-190

in clayey siltstone, B:107

Izu-Bonin forearc, B:102, 104

Ohmachi Seamount, B:187

Site 793, A:338, 340; B:521

Sumisu Rift, B:186, 189

chromite, chemical composition, B:439

chromium

basement, B:434, 437

nickel-magnesium oxide relationship, A:370

Oligocene transition, B:483

chromium/yttrium ratio, Site 793, A:371

chromium/zirconium ratio, basement, B:416

clasts. See andesite clasts; basalt clasts; carbonate bioclasts; pumice clasts; volcanic clasts

clay, classification, A:20

clay, nannofossil

ash beds in, A:154

Site 790, A:145

Site 793, A:323, 326

clay, nannofossil silty

Site 787, A:77

Site 792, A:228

clay, silty

Site 790, A:146

volcanic fragments in, A:153

clay, vitric, X-ray diffraction, A:150

clay, volcaniclastic, depositional process, B:228

clay mineralogy, andesite, B:436, 441, 445

clay minerals, in vein structures, B:198, 205-206

claystone

calcareous component, A:407

color variation, A:329-330

fissility, A:345

magnetic properties, A:84

manganese minerals, B:115

mottled, 787, A:72

Site 791, A:147-148

claystone, nannofossil

basaltic sand grains in, A:157

burrowing, A:335

depositional environment, A:116

physical properties, B:546

Site 787, A:69, 71

Site 788, A:108-110

Site 792, A:235, 239; B:225

trace fossil content, B:225-226

claystone, silty

depositional environment, A:246

laminated, A:77
Site 787, A:73-74

Site 788, A:109

Site 792, A:237

clinoptilolite, A:7; B:522, 524. See also cement, clinoptilolite

clinopyroxene

basement, B:406-407, 419, 444

basement andesite, B:434, 439, 441-442, 446

chemical composition, B:175, 178-179, 433435, 438-441

glass inclusions, B:174-175, 180-181, 183

relict phenocryst composition, B:160

Site 791, B:399

in volcaniclastic sand/sandstone, B:161

coarsening-upward sequence

Site 788, A:116

Sites 790/791, A:155

color banding, Site 793, A:346

color mottling, Site 790, A:145-146

compaction

Pliocene-Pleistocene, A:117

Site 788, A:112, 115-116

conglomerate

depositional environment, A:346-347

depositional processes, B:87, 227, 612

formation microscanner imaging, B:82, 84, 87

Izu-Bonin forearc, B:612

Site 793, A:332-333

X-ray diffraction, A:342

conglomerate, pebble

Site 787, A:73-74

Site 788, A:111

conglomerate, polymictic, Site 792, A:240, 244245

conglomerate, pumiceous

compaction, A:115-116

Site 788, A:109

Site 792, A:240

Site 793, A:336

winnowing, A:157

conglomerate, sandy, depositional environment, A: $246-247$

conglomerate, volcanic

depositional environment, A:248

Site 792, A:235, 237-238

conglomerate, volcanic-lithic, Site 793, A:344; B87

conglomerate, volcaniclastic, hydrothermal alteration, B:419

conglomerate, volcanogenic, petrography, B:406

consolidation, physical property effects, B:544547

convergent margin, circum-Pacific, B:90

copper, B:489

coralline algae, Site 793, B:231-232, 234

Coriolis Trough, isostatic modeling, B:568

cristobalite, X-ray diffraction, A: 148

crystal lithic tuff

Site 791, B:404

Sumisu Rift, B:186, 189-190

Curie temperatures, B:347

dacite

geochemistry, A:265-266

Izu Arc, B:385

Site 792, A:267

trace elements, B:419, 421, 426

dacite clast, Site 792, A:267

debris flow

clasts, B:98-99

Site 792, A:241

volcaniclastic vs. nonvolcanic, B:97

debris flow, submarine, B:97, 99 debris-flow deposits

depositional environment, A:247, 345-346

Izu-Bonin forearc, B:87

Site 793, B:232

Sumisu Rift, B:569

deformation

extensional, B:621

Mariana vs. Izu-Bonin systems, A:5

Site 787, A:69, 73, 79

Site 788, A:112

wet-sediment, A:337

density. See also GRAPE density

calcium carbonate and, A:189

carbonate content and, A:379

dry bulk, B:551-553

gravimetric- vs. pycnometer-determined, B:554

logging data, B:656

olivine diabase intrusion, A:360

Site 787, A:92-93, 200

Site 788, A:122-123, 126

Site 790, A:189, 195, 198-200

Site 791, A:206

Site 792, A:278-279

Site 793, A:379

vs. porosity, B:548

deposition

conglomerate, B:87, 227, 612

sandstone, A:346; B:87, 612

siltstone, B:87, 612

dewatering

structures, Izu-Bonin forearc, A:408; B:205

veinlets, Site 792, A:235

diabase

geochemistry, A:185

petrology, A:177, 181, 184

physical properties, A:200, 379, 386

diabase intrusion

active arc associations, B:417

arc tholeiite affinity, B:442

olivine, A:354, 360-361, 367-369

diagenesis

geochemistry, B:420, 422, 432-433, 435,442

glassy inclusions, A:364

logging data, B:657

petrography, B:432

petrology, A:174, 363

Site 793, B:639-640

diagenesis, B:132

associated demagnetization, A:84

original sediment composition control, B:131132

physical property effects, B:545

pre-Quaternary sandstone, B:468

Site 788, A:110-112

volcaniclastic sand/sandstone, B:129, 131-132

diaspore, X-ray diffraction, A:148

diatoms, amino acid composition. B:537

dikes

petrology, B:392

sandstone, Site 792, A:244

diopside, in volcaniclastic sand/sandstone, B:160

dissolved combined amino acids (DCAA)

as diagenetic indicators, B:531

in interstitial waters, B:534, 536-539

dissolved free amino acids (DFAA)

changes with depth, B:531

in interstitial waters, B:532-537

dynamo, Rikitake model, B:349

earthquakes, magnitude and recurrence interval, B:90-91, 95 
East African rifts, zigzag border fault systems, B:564

El Chichon pumice, anhydrite microphenocrysts, B:451

electrical conductivity, B:604 grain size and, B:607

en echelon ridges, Sumisu Rift, B:642

Eocene/Oligocene boundary, planktonic foraminifers, B:278

epidote, Sumisu Rift, B:186-187, 189

erionite, Site 793, B:134

explosive fountaining, deep-sea, B:373

fallout sedimentation, Site 792, B:220, 225

fault blocks, tilted, B:559

fault gouge

bedding subparallel, B:202

petrology, A:183

X-ray diffraction, A:184, 187

fault zone

Site 792, A:235, 245

Site 793, A:342

faulting. See also border faults; microfaults braided, A:345

dip orientation, A:162

Site 788, A:109, 112, 116

Site 793, A:336

syndepositional, B:559, 562, 646

faulting, half-graben, Izu-Bonin forearc, B:405

faulting, normal

anastomosing and relay pattern, B:559

formation microscanner imaging, B:621

Izu-Bonin forearc, B:584

orthorhombic geometry, B:564

rifting-related, B:634

Sites 790/791, A:129

small-offset, B:559

zigzag pattern, B:564, 566, 634, 642, 647-648

faulting, syn-rift, Izu-Bonin forearc, B:647

feldspar

in hyaloclastic breccia, B:105

Izu-Bonin forearc, B:102

mineralogy, B:111

smectite covering, B:135

felsic clast, petrology, A:183

felsitic grains, stained/etched, B:151

flowage differentiation, Izu-Bonin forearc, B:417

flute casts, in sandstone beds, A:336; B:83

foraminifers

amino acid composition, B:537

in sediment gravity flows, B:94

Site 788, B:137-138

foraminifers, benthic

abundance and preservation, A:24

Assemblages A-D, B:311-313

biostratigraphy, A:80-81, 118, 163, 165-167, 171-172, 251-253, 349, 351; B:288296

biozones, A:165-166; B:288-296

dissolved oxygen inference, A:24, 118; B:294

distribution, B:290-293, 295, 297, 314

Izu-Bonin Arc, B:311-314

Izu-Bonin forearc, B:495

large, $\mathrm{B}: 233,630$

low-oxygen environment, B:228

paleobathymetry, A:23-24; B:288-296, 313314

paleoenvironment, A:81, 84; B:288-296

resedimented, B:87

shallow-water environment, B:646

Site 788, B:290

Site 792, B:290-292

Site 793, B:231-232, 234, 292, 294, 296 stratigraphic hiatuses, A:254

zonation, A:23

foraminifers, planktonic

abundance and preservation, A:23

biostratigraphy, A:80-81, 118, 162-163, 171172, 250-252, 349; B:271-280

Cold Events 1-6 B:281-283

datums, A:24, 167

dissolution effects, B:280-283

distribution, B:274-281

Eocene/Oligocene boundary, B:278

Oligocene/Miocene boundary, B:280

paleoceanography, A:80; B:274

paleoenvironment, A:81

Pliocene/Pleistocene boundary, B:271-272, 281

Site $787, \mathrm{~B}: 272$

Site 788, B:272-273

Site 790, B:273

Site 791, B:273

Site 792, B:273-278

Site 793, B:278-280

stratigraphic hiatuses, A:254; B:272, 275, 277, 281

zonation, A:23

formation factor, A:33; B:547-549

porosity and, B:547-548

Site 787, A:84, 87

Sites 790/791, A:190

Site 792, A:267-269, 271

Site 793, A:371-372

vertical vs. horizontal, B:548-549

formation microscanner (FMS) imaging, B:75, 78

sandstone beds, B:91-93

sedimentary facies, B:607, 612

sensor and data processing, B:604, 607

Site 792, A:289, 295

Site 793, A:389

fractures

extensional, B:205, 206

formation microscanner imaging, B:616-617, $622-623$

gypsum-filled, A:346

Mariana forearc, A:5-6

fracturing, conjugate, Site 787, A:78

frontal-arc highs

Izu-Bonin Arc, B:231, 632

relation to outer-arc high, B:634

Gauss Normal Chronozone

Site 788, A:119

Site 792, A:256

geochemical logging

centroid composition, B:596

data acquisition, B:653-654

data reduction, B:654-656

high gamma ray horizons, A:208

in-situ measurements, A:307-308

Izu-Bonin Arc, B:653-676

lithologic correlation, B:594, 656-657

lithostratigraphic correlation, A:206-208, 306

oxide percentages, B:656-657, 662-663, 668669, 674-676

seismic correlation, B:579-580, 582-583, 586-587, 590-591

Site 791, A:202-203, 206-209; B:660-663

Site 792, A:289, 296-301; B:598, 664-669

Site 793, A:389-395; B:599, 670-676

vs. core measurements, B:593-596

geochemical logging tool (GLT) string, B:654

geochemistry, hydrothermal effects, B:497

geochemistry, fluid. See also interstitial-water chemistry analytical methods, A:32-33

seawater interaction effects, A:89

Site 787, A:85, 87-88

Site 788, A:122

Sites 790/791, A:187-188

Site 792, A:268-270

Site 793, A:371-378

geochemistry, igneous

alteration effects, B:419

basement, A:270-271, 369

bimodal, B:639

calc-alkaline affinity, A:266

high-field strength elements, A:266

least-squares mixing, B:407, 409, 414

olivine diabase intrusion, A:367-369

pre-rift vs. rift, B:392

Sites 788/789, A:119-121

Sites 790/791, A:184-185

Site 792, A:265-267; B:419, 421, 457-463

Site 793, B:407-417

volcanic pebbles, A:369

geochemistry, inorganic. See also geochemical logging

core vs. log measurements, B:593-596

geochemistry, sediment

analytical methods, A:34-35

Site 787, A:85; B:472, 475, 490, 494

Site 788 A:122; B:386-387, 472, 475, 490, 494

Site 790 A:186-187; B:472, 475, 478, 490, 494

Site 791, A:186-187; B:391-392, 472-473, 475-476, 478-479, 490-491, 494

Site 792, A:269; B:473, 476, 478-479, 491492, 494-495

Site 793, A:378-379; B:473, 476-479, 492, 495

geomagnetic field, intensity oscillations, B:349, 351

geomagnetic pole, virtual (VGP), B:342-351

geophysical logging

data reprocessing, A:41

Izu-Bonin forearc, B:608-611

lithostratigraphic correlation, A:306, 308-309, 401-402

measurements, A:37-39

operations, A:228, 286-288, 388, 395, 402

Site 792, A:223, 289ff; B:610-611

Site 793, A:396-401; B:608-609

geothermal gradient, B:191

Gilbert Normal Chronozone, Site 788, A:119

glass

Site 790, A:141

sulfur content and alteration of, B:451

glass, brown, B:45

alteration, B:136

Site 793, B:152

glass, intermediate-to-mafic, dissolution, B:129

glass, rhyolitic, alteration, B:129

glass, unmixed, Izu-Bonin forearc, B:175, 182

glass, vitric, B:141, 154

glass, volcanic

alteration, B:152

analytical methods, B:509-510

bubble-wall type, B:45, 49

chemistry, B:34, 41, 59-60

color, B:140, 143-145, 147-150

diagenetic alteration, B:126

felsic input, B:143

hydration, B:512-513

hydrothermal alteration, B:105, 107

INA analyses, B:457-458

isolation of, B:457 
Izu-Bonin forearc, B:101

juvenile shard, B:514

magma composition, B:505

morphology, B:49

oxide chemistry, B:63, 70

oxide stratigraphy, B:510-516, 518-524

Site 793, A:334

size range and morphology, B:509

systematic weight loss, B:510-513

glass inclusions

analytical technique, B:172, 174

in basaltic mousse, B:391, 402

chemical composition, B:174-175, 180-181

crystallization from unevolved magma, B:175

magma mixing and, B:171, 175

with microglobules, B:174

occurrence, B:174-175, 182

in pyroxene-plagioclase phenocrysts, B:171181

Site 793, A:360, 364

glauconite

depositional environment, A:116

Site $788, \mathrm{~B}: 228$

vs. celadonite, A:114

glauconite-celadonite group, chemical composition, B:436

glutamic acid, decomposition, B:538

glycine, Sumisu Rift, B:537

graded beds

inverse, B:87

Site 790, A:144, 149

Site 792, A:239

grain size

conglomerates, B:84

physical properties and, A:278

pumice beds, B:8-10, 19

resistivity and, B:78

volcanic ash layers, B:27, 34

GRAPE density

carbonate content and, A:379

Site 787, A:91, 93, 200

Site 788, A:122-123, 126

Site 790, A:189

Site 791, A:199-200, 206

Site 792, A:278-279

gravel

Site 788, A:415

Site 790, A:141, 144

gravel, pebble, Site 788, A:104, 106

gravel, pumiceous

depositional environment, A:117, 325

role of volcanism in, A:159

scoriaceous, A:71; B:640

Site 787, A:71

Site 788, A:104, 106, 109

Site 790, A:140-141, 144, 147

Site 793, A:323, 327

transport mechanisms, A:158-159

Guam

Winnowing, A:157

Alutom Formation, B:632

volcanic highs, B:630

gypsum

in clayey siltstone, B:107

precipitation, A:378; B:521

Site 792, A:270

X-ray diffraction, A:185

Hachijo Island

pyroclastics, B:49

volcaniclastic sand, B:144

Hahajima Island

Nummulites boninensis, B:233 volcanic highs, B:630

half grabens, sediment-filled. B:634

heat flow

rift basins, B:643

Site 792, A:285-286

hemipelagic sediment

analytical methods, B:487

authigenic components, B:497-498

biogenic components, B:489, 495, 497

deposition, B:213, 227-229

geochemistry, B:489-499

hydrothermal effect, B:497

mass-flow deposition, B:225, 229

sedimentary environment, B:498-499

Site 793, A:344

source, B:489, 497-499, 515

terrigenous component, B:497

velocity-porosity relations, B:547

hemipelagites, sedimentation rates, B:7-8

heulandite, Site 793, B:134, 438-439, 445, 523

high-field-strength elements (HFSE)

olivine diabase intrusions, A:369

tephras, B:461, 463

Hokuroku Basin, Kuroko deposits, B:643

hornblende

Site 792, B:168

in volcaniclastic sand/sandstone, B:160

hyaloclastite, Site 792, A:263-264

hydraulic sorting, turbiditic sands and sandstones, B:468

hydrocarbon gases, Sites 790/791, A:187

hydrodynamism, Site 788, B:218, 228

hydrogen, Site 791, A:203, 205, 211-215

hydrothermal circulation. See also alteration, hydrothermal

in backarc rift basins, B:555, 557

Site 792, A:248

Sumisu Rift, B:190, 642-644, 646

igneous petrology

basement, A:262-263, 362-367

breccias, B:406

lava, A:263-265

Sites 790/791, A:174, 176-177, 179, 181-184

units, A:176-184; B:391, 419

volcanic clasts, A:361-362

igneous rock

physical properties, A:388

Pleistocene, A:415, 418

illite/smectite, mixed-layer, Site 793, B:524

ilmenite, Site 792, A:264, 268

immiscible liquids

in glass inclusions, B:175

in plagioclase phenocrysts, B:174

impedance, Site 792, A:308

incompatible elements

fractional crystallization effects, B:394

in rhyolite pumice, A:120-121; B:386-388

Site 792, A:266

subduction-related, A:120

Indian Ocean SW, virtual geomagnetic pole (VGP) migration, B:346

interstitial-water chemistry

advection effects, B:525, 527

alteration effects, A:372, 378; B:125, 520521,527

concentration gradients, B:519-521, 525, 527

controls on, B:520

diagenetic quiet zone, B:527

hydrothermal circulation, B:107

mineralogical comparison, B:104-105

Site 787, A:89; B:522-523

Site 788, A:122-123; B:522-523
Site 790, B:522-523

Sites 790/791, A:187-188, 194

Site 792, A:222, 276-277

Site 793, A:316, 372-373; B:522-523

thermal gradients, B:527

thermodynamic aspects, B:519-528

iron

basement, B:433-434

enrichment mechanisms, A:367, 369

logging data, A:206, 208

in manganese deposits, B:122-123

Site 787, A:88

iron oxide

ash layers, B:516

basement, A:369-370; B:440, 445, 447

core vs. $\log$ measurements, B:593-594

logging data, B:656

magnesium oxide correlation, A:266, 269

in pelagic/hemipelagic sediments, B:501

sinks., B:521

iron oxide/aluminum oxide ratio, Izu-Bonin forearc, B:187, 189

iron oxide/magnesium oxide ratio, B:409, 423,470

iron oxide/silica ratio, Sumisu Rift, B:187, 190

iron-titanium oxide, Site 791, B:399

island-arc basalt (IAB), associated magmas, B:383

island-arc tholeiite, trace elements, B:479

island arcs, geochemical signature, B:393

isostatic rebound, Sumisu Rift, B:566-567, 644

isothermal remanent magnetization (IRM), B:333, 342

iterative, nonhierarchical cluster analysis (INCA), Izu-Bonin forearc, B:600-601

Izu Arc, volcanism, B:386-388, 393-395

Izu-Bonin Arc

accretion to south-central Honshu, B:634

arc margin, B:557, 559

ash layers, B:27-39

backarc, A:415-418; B:185

basement, A:51-52; B:231

bathymetry, A:52, 408; B:172, 272, 488, 641

benthic foraminifers, B:311-314

evolution, B:479

forearc, A:51-52

frontal-arc highs, B:632

geochemical evolution, B:481-483

geochemical logging, B:653-676

geological setting, B:3, 113, 185, 231, 488489

glass shards, B:510-516

incompatible elements, A:120

Kyushu-Palau Ridge connection, B:206

lithospheric extension, B:555

lithostratigraphy, B:26, 507

manganese deposits, B:114-122

massif width, B:634

multichannel seismic survey, A:56

Oligocene, B:417, 419

Oligocene-Miocene strata, A:407

paleobathymetry, B:313-314

Pliocene-Quaternary strata, A:409

pumice, B: 18

quartz keratophyres, A:121

rift basins, B:508, 555

rift-flank succession, B:508

rifting, A:9, 418; B:508, 647

stress field orientation, B:619-620, 624

structural features, A:409

submarine shield volcano, B:647

tephra, B:42

topography, B:355

uplift, A:418 
volcaniclastic sand/sandstone, B:145, 147, 470-479

volcaniclastics, B:155, 158-160, 483

volcanism, B:160-161, 629, 646

Izu-Bonin arc-trench system, bathymetry, A:65, 99, 130, 224, 317; B:24, 48, 140, 238, 406,638

Izu-Bonin backarc

lithostratigraphy, B:26

pumice beds, B:6-8

volcaniclastics, B:155, 158-160

Izu-Bonin forearc

alteration mineralogy, B:105-106

andesite, B:436

basement, A:6; B:677, 679

basin evolution, A:408; B:75, 113-114, 231, 603, 632-633

calcareous nannofossils, B:239-258, 495

chemostratigraphy, B:594, 596

diabase intrusive, B:417

faulting, B:584, 647-648

geochemical logging, B:593-596

geological setting, B:677

geomorphological provinces, B:648

geothermal gradient, B:191

hydrothermal alteration, B:101-107

intersite correlation, A:407-413

lithospheric extension, B:583

lithostratigraphy, B:25, 76-78, 603-604

microstructures, A:64

outer-arc basement high, A:5

paleobathymetry, B:299-301

paleoceanography, B:280-283

paleoenvironment, B:233, 290-298

petrogenesis, B:422, 426

petrography, B:105

plate rotations, A:64, 318

pumice beds, B:9

rare earth elements, B:408

rift-basin development, B:405

sandstone beds, B:87-90

sedimentary structures, B:607, 614, 618-620

sedimentation, A:318; B:76, 78, 95, 498-499, $584,603,647$

seismic reflection profiling, B:634, 636-637

seismic stratigraphy, A:66; B:557, 575, 579ff

structural evolution, B:640, 647

tectonic evolution, A:5-6

tephra, B:49, 51,59-63,68

terrane origin and evolution, A:224, 318

thermal history, B:131

uplift/subsidence history, A:64, 223; B:298

vein structures, B:195-206

vertical displacement history, A:318

vertical motion history, B:640

volcanic history, A:6; B:285

volcaniclastics, B:75, 78-90, 155, 158-160, 171-175

volcanics, B:421-422

Izu-Bonin-Mariana Arc

backarc, B:636, 638-639

evolution, A:6, 8-9

extension structures, B:634

faulting, B:642

forearc, B:634

frontal arc, B:630-631

rifting, B:627, 629, 632-636, 640, 642-644, 646

sedimentation, B:634, 637, 639-640

volcanism, B:629-632, 639-640, 642, 648

Izu-Bonin region

bathymetry, B:286, 312

pumice, B:8-11 volcaniclastic sediment, B:543-549

Izu-Bonin rift, tectonic setting, A:9

Izu-Ogasawara Arc, tephra, B:457-463

Izu-Palau Arc system, evolution, B:362-364, 36\& 368

Japan Arc SW, and Japan Sea opening, B:364

Japan forearc, Oligocene, B:364

Japan Sea, basin opening, B:364

Japan Trench

argillaceous sediment, B:487

vein structures, B:195

Juan de Fuca Ridge, basaltic glass, B:510

Kaikata Seamount, todorokite, A:116

Kilauea lava fractionation factor, B:451-452

Korobasaga Volcanic Group (KVG), geochemistry, B:395

Kotori Volcano

location, B:557

as sediment source, B:569

Kozushima Island, white tephra, B:49

Kurasaki Volcano, marine tephra, B:42

Kurasaki volcanics, Site 792, B:63

Kyushu-Palau Ridge, Izu-Bonin island arc connection, B:206

lamination

normal-to-diffuse transition, A:87

sandstone beds, B:84

lamination, cross

formation microscanner imaging, B:618

ripple-scale, B:91-93, 95, 612

lamination, parallel, in pumiceous sand beds, A: 148

lanthanum/ytterbium ratio, B:479, 484

lapilli tuff

geochemistry, A:185

petrology, A:174, 181-182, 184

physical properties, A:200

Site 787, A:69, 74

Site 790, A:129

sonic velocity, A:93

large-ion-lithophile (LIL) elements, tephras, B: 461,463

Lao Basin

arc volcanism, B:394-395

forearc spreading, B:647

lava

barium-poor, B:391

groundmass texture, A:262

petrology, B:389-391

source, B:642, 646

stratigraphy, A:263-265

tholeiitic differentiation, B:390

lava, andesitic, petrology, A:363-365, 367

lava, basement

petrology, A:222; B:389-391

phenocryst assemblages, B:406

lava, boninitic, petrology, A:365, 367

lava, calc-alkaline, Site 792, B:422

lava, hyaloclastic, nonexplosive emplacement, B:373

lava, porphyritic

mineralogy, B:111

Site 793, A:347

lead isotopes, basement, B:422, 426-428

Leg 125

objectives, B:627

volcanic rock, B:467

volcaniclastic sandstone, B:483

Leg 126

bathymetry, A:43-44; B:4 chronostratigraphy, B:249, 251

navigation, A:42

objectives, A:10; B:101, 140, 209, 353, 487, 505,627

seismic reflection profiling, A:43-46

limestone

depositional environment, B:233

Site 793, B:231-233

lithification

sandy sediments, B:125-127

Site 788, A:107-108, 110-112

volcaniclastic sandstone, B:131

lithium

Site 787, A:88

Sites 790/791, A:187

lithosphere, linear zone of weakness, B:564, 570571, 647-648

lithospheric extension

mechanisms, B:555

onset, B:583

lithostratigraphy

basement, A:180

composite section, A:140, 230-231

composite succession, A:156

correlation, Sites 790 and 791, A:151-152, 155

depositional environment, A:115-117, 155$160,244-248,325-328,342,344-348$

facies associations, A:336, 338

gravel-to-conglomerate transition, A:108

hiatuses, A:228; B:579-580

Izu-Bonin forearc, B:76-78

lithologic summary, A:157

lithologic Unit I, A:63, 68-69, 75, 77, 104, 106-108, 129, 140-141, 144-148, 228-229, 244, 322, 325-328, 338, 415, 418; B:4, 6, 8, 114, 210, 215, 217-220, 508-509, 533, 559, 576, 579, 581-582

lithologic Unit II, A:63, 69, 77, 108-110, 130, 145-148, 155, 157, 229, 244-245, $328,415,418 ; \mathrm{B}: 6,213,215,217$, 220-225, 229, 506, 509, 533, 559, $576,579-580,582,656-657$

lithologic Unit III, A:63-64, 69, 77-78, 229, 235, 246, 328-329, 338, 342, 344; B:6, 225, 533, 576, 580, 582-583, 657

lithologic Unit IV, A:63-64, 71-74, 78-79, 235, 237, 246-248, 329-332, 338, 344-345; B:115, 227, 580, 583, 657

lithologic Unit V, A:237-239, 248, 332-338, 340, 345-348; B:115, 227, 580, 583, 657

lithologic Unit VI, A:240, 337-338, 348; B:583, 657

lithologic Unit VII, B:583

logging correlation, A:401-402; B:593-596

magnetic susceptibility and, A:418

physical property correlation, A:379, 386, 388

seismic correlation, A:67, 103, 129-130, 132, $135,137,226,319$; B:576-585, 587$588,590-591$

Site 787, A:71, 410; B76-78, 282

Site 788, A:105, 415; B:16, 157, 282, 565, 645

Site 790, B:282, 565, 645

Sites 790/791, A:157

Site 791, B:282, 565, 645

Site 792, A:410; B76-78, 282

Site 793, A:323-324, 411; B:17, 76-78, 282

tripartite beds, A:144-145, 151-152

unconformity, A:117

logging. See geochemical logging; geophysical logging 
Maemong Limestone (Guam), insoluble residues, B:639

magma

backarc vs. arc, B:394

evolution, B:461

pre- vs. syn-rift, B:387, 395, 398

rift vs. arc, B:383

sources, B:393-394

trench vs. arc, B:422, 426

magma, boninitic, genesis, B:630

magma, silicic, backarc rifting and composition of, B:482-483

magma, tholeiitic basaltic, pressure compensation level, B:371

magma eruption

andesitic, B:513

explosive rhyolitic, B:513

rate during incipient rifting, B:393

sequences $14, \mathrm{~B}: 482$

magma mixing

eruptive, B: 144

glass inclusions and, B:171, 175

magmatism

with arc volcanism, B:483

backarc, B:566

rifting-related, B:483

syn-rift, B:442

magnesium

alteration effects, A:269, 378

basement, B:434, 437

Miocene/Oligocene boundary gradient, B:519520

Site 787, A:88

Site 793, A:372

magnesium/iron ratio, of smectite, B:521

magnesium oxide

aluminum oxide correlation, A:370

ash layers, B:516

chemostratigraphic variation, B:419

chromium-nickel relationship, A:370

iron oxide correlation, A:266, 269

Oligocene transition, B:483

in pelagic/hemipelagic sediments, B:497, 499, 501

sinks, B:521

vs. silica, B:471

magnesium oxide/silica ratio

basement, B:423

Site 793, A:370

magnetic anomalies, B:359

asymmetric accretion, B:636, 638-693

magnetic polarity reversals, dipole field component, B:351

magnetic properties, A:43; B:354-357. See also natural remanent magnetization (NRM)

AF demagnetization, B:355-357

AF magnetization, B:347, 351

altered vs. unaltered materials, B:333

anhysteretic remanent magnetization (ARM), A:169; B:342, 347, 351

azimuthally oriented directions, B:353, 357, 359,362-363

basaltic mousse, B:371-376

Brunhes/Matuyama reversal, B:342-351

conglomerate test, B:355, 358

declination, B:357, 359, 363-366

demagnetization behavior, B:343, 346-347

directional behavior, B:342

inclination, B:355, 357

intensity, B:342-345, 347, 349, 354-355

lithostratigraphic correlation, A:418

measurement problems, A:261

median demagnetizing field (MDF), B:372
Site 787, A:82-85, 87; B:353-369

Site 788, A:118-119ff

Site 790, A:169-174ff; B:334, 340

Site 791, A:169-174ff; B:333, 335, 337, 339340

Site 792, A:222-223, 254-261ff; B:335-340, 353-369

Site 793, A:352-354ff; B:333, 336, 340, 353 369

soft-component magnetization, B:357, 359, 362

susceptibility, A:146, 148

thermal demagnetization, B:355, 357

thermal magnetization, B:344

thermal remanent magnetization (TRM), B:373

virtual geomagnetic pole (VGP), B:342-351

viscous remanent magnetization (VRM), B: 347,357

magnetite, basement, B:419, 436

magnetostratigraphy, B:238

biostratigraphic correlation, A:256-257; B:257

Brunhes/Matayuma reversal record, A:172, $174,177,257-261,352,354$

Chronozone C5, B:249

Chronozone C5-C11, A:352

Chronozone C5A, B:249

Chronozone C8N, A:257

Chronozone C9Na, A:257

polarity reversals, B:253

remagnetization, A:256, 261

Site 787, A:84, 257,262

Site 788, A:118-119

Site 790, A:169-170

Site 791, A:170-174

Site 792, A:254-257

Site 793, A:352

manganates, Izu-Bonin forearc, B:115, 121

manganese

in claystone, B:114-117

depositional environment, B:117

genetic classification, B:113

hydrothermal, B:113

Izu-Bonin Arc, B:114-117

mineralogy and chemistry, B:115, 117, 122123

Site 787, A:88

Site 788, A:122

Sumisu Rift, B:114

manganese oxide

forearc seamount B:191

in hemipelagic sediments, B:497, 499

origin, A:117; B:497-498

on pumice pebble, A:107

mantle, sulfur isotope composition, B:449

Mariana Arc

rifting, B:557

volcanism, B:463, 648

Mariana basin, backarc spreading, A:5

Mariana forearc, evolution, A:5-6, 8

Mariana Islands, declination anomalies, B:353

Mariana Trench, leucocratic plutonic rocks, B:630

mass spectrometry, inductively coupled (ICPMS), B:469, 477-478

mass wasting, Izu-Bonin-Mariana Arc, B:640

Matuyama Chronozone, Site 792, A:255-256

mean blocking temperature (MBT), basaltic mousse, B:372

metamorphism

basalts, A:174

heat source, B:191

Izu-Bonin forearc, B:189-191

origin, B:189-190 prehnite-pumpellyite facies, B:189

tectonics and diversity in, B:185

timing of, B:190-191

metavolcanic rock, origin, B:190

microbreccia, volcanic, Site 793, A:337-338

microfaults

anastomosing, A:345

extensional, A:77, 246, 408

Site 793, A:334, 336

microfaults, normal, Site 793, A:334

microfractures, Site 788, A:106-109

microstructures

Izu-Bonin forearc, A:224, 318

Site 793, B:618

mid-ocean-ridge basalt (MORB)

associated magmas, B:383

E-, Sumisu Rift, B:396, 398

$\mathrm{N}-$, trace elements, B:477

sulfur isotopes, B:449

Middle America Trench, vein structures, B:195, 205

Minami-Somisu caldera, pumice, A:121; B:10

Minamizaki limestone

Bonin Islands, B:632

foraminifers, B:233

mineralogy

authigenic phases, A:373

chemical-physical correlation, B:104-105

$d$-spacing, A:150

igneous, B:440-442

secondary, B:436-439

Site 793, B:432-436

Sumisu Rift, B:187-188

thermodynamic correlation, B:524-525

in volcaniclastic sand/sandstone, B:161, 163

Miocene/Oligocene boundary, interstitial-water gradients, B:519-520

Miura-Boso peninsulas, vein structures, B:195, 206

Miyakejima Island, pyroclastics, B:49

Miyojinsho volcano, pumice, B:514

montmorillonite

Sumisu Rift, B:538

in vein structures, B:206

mordenite, Site 793, B:134

mud, hemipelagic, incompatible elements, B:388

mud, nannofossil sandy, X-ray diffraction, A:148

mud clasts, in sandstone beds, B:85

mudrock, Izu-Bonin forearc, B:80, 612

mudstone, radiolarian-dominant, B:201

mudstone, sandy

depositional environment, A:244-245

plastic folding, A:76

Site 792, A:229, 237

Site 793, A:332

Nankai, thrusting, subduction-related, B:634

nannofossils, calcareous

abundance and preservation, A:21, 23; B:241242,246

Assemblages 1-5, B:239-240, 242

biostratigraphy, A:79-81, 117-118, 160-162, $167,171-172,248-250,252,347-$ 349; B:257

datums, B:256

distribution, B: 240

Gartner zonation compared, B:246

lithologic correlation, B:245-246

magnetostratigraphic correlation, B:249, 251

Matsuoka and Okada assemblages compared, B:242-244, 246

Oligocene/Miocene boundary, A:21; B:247

Oligocene-Miocene, B:246-247, 249 
Pleistocene, B:239-246

Pliocene/Pleistocene boundary, A:21

Site 787, B:248-249

Site 790, B:240

Site 791, B:241

Site 792, B:242, 250, 254-256

Site 793, B:252-253

stratigraphic hiatuses, A:254; B:247

Sumisu Rift, B:240-242

synsedimentary reworking, B:241

taxonomic classification, B:237, 239

zonation, A:21

natural gamma spectrometry

Site 792, A:289

Site 793, A:389

natural remanent magnetization (NRM), B:333, 342-345

anhysteretic remanent magnetization (ARM) ratio, $B: 343,347-348,352$

basaltic mousse, B:372, 374-376

isothermal remanent magnetization ratio, B:333-339, 343, 347-348, 372

oscillating behavior, B:346, 349, 351

signal-to-noise ratio and, B:347

stable component direction, B:354-355

vein structures, B:204-205

neodymium isotopes

arc vs. rift, B:398

basement, B:421-422, 426-427

in glass shards, $\mathrm{B}: 461$

in volcanic rocks, B:391, 396

neodymium/zirconium ratio, basement, B:416

New Guinea Plate N, B:630

nickel, B:489

chromium-magnesium oxide relationship, A; 370

nickel/zirconium ratio, basement, B:416

Niijima Island, white tephra, B:49

Nishinoshima Island, todorokite, A:116

Nishinoshima Trough

rift morphology, B:185

sediment-filled half graben, B:634

Nishiyama Mudstone Formation (Japan), vein structures, B:205

nitrogen, total

Site $787, \mathrm{~A}: 85,88$

Site 788, A:122

Sites 790/791, A:186-187, 191-193

Site 792, A:268, 272-276

Site 793, A:374-378

nucleation kinetics, B:525

ocean-island arcs, geochemical evolution, B:479, 483

ocean-island basalt (OIB), sulfur isotopes, B:449

Oceanic Formation (Barbados), vein structures, B:205

Ohmachi Seamount

carbonate bioclasts, B:233

geological setting, B:185, 231

petrography, B:186-187

prehnite-pumpellyite metamorphism, B:189192

Oligocene/Miocene boundary

calcareous nannofossils, A:21; B:247

planktonic foraminifers, B:280

olivine

in basaltic mousse, B:391

basement, B:389, 399, 406-407, 439

chemical composition, B:162-163

in Sumisu Rift basalts, B:396

in volcaniclastic sand/sandstone, B:158, 160

olivine diabase intrusion geochemistry, A:367-369

petrography, A:360-361

petrology, A:354, 360

Onnagawa Formation, vein structures, B:205

ooze, nannofossil, Site 787, A:77

opal-CT

formation conditions, B:525, 527

as silica sink, B:525

opaque minerals, B:140

diagenetic, B:126

ophiolites, supra-subduction zone, A:6

ornithine, Sumisu Rift, B:538

orthoclase, andesite-plagioclase composition, B:443

orthopyroxene

basement, B:406-407, 419, 435, 439, 444

chemical composition, B:176-177, 438

glass inclusions, B:182

phenocrysts, A:263, 266

posteruptive alteration, A:262

in volcaniclastic sand/sandstone, B:158, 160

Oshima Island, pyroclastics, B:49

outer-arc high $(\mathrm{OAH})$

Izu-Bonin Arc, B:231

reflection profiling, B:631

relation to frontal-arc highs, B:634

oxygen fugacity, sulfur content and, B:451

$P$-wave velocity

Site 788, A:126

Site 792, A:285

Pacific Ocean W

boninite, B:426

boninite series volcanics, B:422

tectonics, B:139, 556

Palau Islands, declination, B:353, 359, 363

Palau-Kyushu Ridge

leucocratic plutonic rocks, B:630

tuffs and chalks, B:467

volcanic basement, B:467

volcaniclastics, B:632

volcanism, B:629, 639

paleocurrents

analytical methods, B:91-93

directions, B:612

of sediment gravity flows, B:233

paleoenvironment, Izu-Bonin arc system, B:288298

paleolatitude, B:364

magnetic inclination and, B:355, 357

Site 787, A:84

Site 792, A:257

Site 793, A:352, 354

Parece Vela Basin

backarc spreading, A:5; B:636

volcanism, B:648

pelagic sediment

analytical methods, B:487

authigenic components, B:497-498

biogenic components, B:489, 495, 497

geochemistry, B:487, 489-499

Oligocene, B:632

redeposition, B:646

sedimentary environment, B:498-499

source area, B:489, 497-499

terrigenous component, B:497

Philippine Basin W, magnetic anomalies, B:359

Philippine Islands, northward motion, A:9

Philippine Sea

active plate boundaries, A:6; B:628

andesite, A:271

arc volcanism, B:47

basalt, A:271 diagenetic trends, $\mathrm{B}: 132$

magnetics, B:353, 364-365

relict spreading centers, A:6; B:628

tectonic features, B:354

Philippine Sea Plate

rotation, A:9; B:75, 364, 367, 603

subduction, B:630

tectonic evolution, B:362-364, 366-367

phillipsite, B:524. See also cement, phillipsite

phosphate, in volcaniclastic sandstone, B:483

phosphate/yttrium ratio, basement, B:426

phosphorus, basement, B:407, 419

phosphorus/zirconium ratio, basement, B:416

photoelectric effect, predicted vs. actual, A:41

physical properties

analytical methods, A:35-37

chemical-mineralogical correlation, A:200

depositional processes affecting, B:544-548

lithologic correlation, A:379

lithostratigraphic correlation, A:92

logging data, B:607

mineralogical correlation, B: 104-105

seismic correlation, B:579-583, 586-587, 590-591

Site 787, A:88-94

Site 788, A:122-123, 125; B:544-549

Site 790, A:188-202ff

Site 791, A:188-202ff; B:544-549

Site 792, A:223, 271, 276, 278-286; B:544 549

Site 793, A:316, 379-388; B:544-549

unconsolidated-to-lithified transition, B:545

volcaniclastic sediments, B:543-549

pigeonite, in volcaniclastic sand/sandstone, B:160

Plagioclase

basement, B:389, 399, 406-407, 419

basement andesite, B:434-436, 439-442, 446447

chemical composition, B:176-179, 432-433

glass inclusions, B:171-175, 183

stained/etched, B:151, 154

Plagioclase feldspar

dissolution, B:138

in volcaniclastic sands, B:140

plant debris, carbonaceous, Site 792, A:243

plate rotation, Izu-Bonin forearc, A:224

pleochroism, in pumpellyite replacement, B:186

Pliocene/Pleistocene boundary

calcareous nannofossils, A:21

planktonic foraminifers, B:271-272, 281

plutonic rock, leucocratic, B:630

pore pressure, in fracture formation, B:205

porosity

cementation effects, B:545

formation factor and, B:547-548

indurated pumice, B:126

interparticle, A:334

lithostratigraphic correlation, A:278-279

logging data, B:656

olivine diabase intrusion, A:360

properties affecting, B:551

pumice conglomerates, B:546

Site 787, A:89, 91-92

Site 788, A:123, 126

Site 790, A:189

Site 791, A:199-200

Site 793, A:379

volcaniclastic sandstones, B:126

vs. density, B:548

porosity/cement relationship, volcaniclastic sandstone, B:129

porosity/velocity relations, B:545-546

porphyritic-andesitic flow, Site 792, B:439 
potash, vs. silica, B:470

potassium

in glass inclusions, B: 173

logging data, A:216-220, 289; B:655

Miocene/Oligocene boundary gradient, B:519520

Site $787, \mathrm{~A}: 88$

Site 790, A:187

thermodynamics, B:523-524

in volcaniclastic sand/sandstones, B:470

potassium-argon dating, B:677-680

potassium oxide

ash layers, B:515

basement, A:369

in glass shards, B:512

Site 792, A:266

tephra layers, B:59-60

in volcanic glass, B:34, 63

potassium oxide-silica relationship

pumice beds, B:12-14

tephras, B:40, 62, 74

prehnite. See also metamorphism, prehnite-pumpellyite facies

chemical composition, B:189, 191

Izu-Bonin forearc, B:105, 107

Ohmachi Seamount, B:186-187, 189

pressure compensation level (PCL), tholeiitic basaltic magmas, B:371

proto-remnant arc

arc volcanism on, B:395

Sumisu Rift, B:559

pumice

ages, B:7-9

alteration, B:136-137

andesite-to-rhyolite transition, B:395

bed thickness, A:116

Beds I-V, B:5-6

chemistry, B:7, 9, 20-21

compaction, B:129, 136-137

dissolution rate, $\mathrm{B}: 525$

distribution, B:11

dry-bulk density, B:553

eruption episodes, A:116

geochemistry, B:385, 387-388

grain types, B:7-11, 19

indurated, B:126

Izu-Bonin Arc, B:44

lithologies, B:9

low-alkali tholeiite series, B:9

major oxides, B:12-14

origin and deposition, B:9-11

oxide stratigraphy, B:512

petrography, B:7

petrology, B:396, 398

physical properties, A:199

Pliocene-Quaternary unconformity, B:11, $386,508,570$

pressure welding, A:112

Site 793, A:326, 336

Sumisu Rift, B:408

textures, B:6-7

thickness, B:4

trace elements, B:481

upward-coarsening sequence, B:482

woody fragments, B:151

pumice, anhydrous, B:389

pumice, dacite, B:387-388

pumice, rhyolite, A:120-121; B:386-388

pumice, silicic, B:386-388

pumice clasts

depositional mechanisms, A:117

Site 788, A:106-107, 109

Site 793, A:325, 331
X-ray diffraction, A:148

pumice conglomerate, physical properties, B:546

pumice gravel

manganese coating, B:114

petrography, B:126

Sumisu Rift, B:285

pumice pebbles, Site 790, A:155

pumiceous sediment

consolidation behavior, B:546-547

current control, B:147

lithification, B:131

temperature and age, B:528

pumpellyite

Ohmachi Seamount, B:186

Sumisu Rift, B:186-187

pyrite

sulfur and, A:208

$\mathrm{X}$-ray diffraction, A:150

pyroclastics

classification, A:20-21

grain morphoscopy, B:225

redeposition, B:646

Sites 790/791, A:158

Site 792, B:221-223

pyroxene

calcium content, B:164-168

cockscomb texture, B:140

in volcaniclastic sand/sandstone, B:161

quartz

basement andesite, B:439

Site 792, A:264

in volcaniclastic sands, B:140

radioactivity, logging data, B:655

radiolarians, $\mathrm{B}: 321-330$

abundance, A:24-25

biostratigraphy, A:26-28, 80-81, 167-168, $171-172,252-254,351$

Site 787, B:322-323

Site 790, B:322-324

Site 791, B:322, 325-326

Site 792, B:322-323, 327-329

Site 793, B:324, 330

stratigraphic hiatuses, A:254

in vein structures, B:198, 201

zonation, A:24; B:321-322

random Cantor dusts, in calculating bed thickness frequency, B:88

rare earth elements

basement lavas, B:398, 417

geochemical characteristics, B:489

LREE depletion history, B:479, 483

neodymium isotopes and redistribution of, B:421-422

in pelagic/hemipelagic sediments, B:497-499, 502

pumice, B:387-388

slab component, B:483

temporal distribution, B:461, 480

tephras, B:461, 464

volcaniclastic sand/sandstones, B:478-479, 482

zigzag patterns, B:498

resistivity, pore-water

Site 787, A:87

Sites 790/791, A:190

Site 792, A:271

Site 793, A:372

resistivity, sediment, A:33-34; B:547, 607

grain size correlation, $\mathrm{B}: 78$

Site 787, A:84-85, 87

Sites 790/791, A:186. 190
Site 792, A:267-268, 271

Site 793, A:371-372

resistivity $\operatorname{logs}$

Site 792, A:289

Site 793, A:389

rhyolite

arc vs. rift, B:393, 395-396

geochemistry, B:393, 395-396, 401, 419

Izu Arc, B:385

origin magmas, B:512

petrology, B:392-393

source, B:393

trace elements, $B: 421$

Types A and B, A:121

rhyolite dome, hydrothermal deposits, B:642-643

rift, failed, Izu-Bonin forearc, B:196, 206

rift flank uplift, Sumisu Rift, B:559, 566, 642, 644, 646

rift volcanism

across transfer zones, B:642

forearc, B:405

rifting. See also arc rifting

initiation, A:418; B:386

interarc, B:482

Izu-Bonin arc, A:9

Izu-Bonin-Mariana Arc, B:557

uplift, A:418

rubidium, in basement basalts, A:184

Ryuku Arc

ash layers, B:463

volcanic history, B:68

Saipan

declination anomalies, B:359, 362

leucocratic plutonic rocks, B:630

volcanic highs, B:630

samarium/zirconium ratio, basement, B:416

sand

physical properties, A:91-93

Site 791, A:147

sand, basaltic, in nannofossil claystones, A:157

sand, black

Site 790, A:145

Site 791, A:159

sand, pebbly

Site 790, A:144

Site 791, A:147, 158

sand, pumiceous

depositional environment, A:326

parallel laminations, A:149

Site 790, A:144

Site 791, A:158

Site 793, A:329

sand, vitric

depositional environment, A:326

felsic and mafic components, A:228-229

graded beds, A:328-329

Site 788, A:107

Site 791, A:160

Site 793, A:323, 325, 327

X-ray diffraction, A:148, 150

sand, volcaniclastic, B:140-144

detrital modes, B:142-144

geochemistry, B:160

grain dissolution and alteration, B:129

grain types, B:140-141

heterogeneity, B:147, 153

Izu-Bonin region, B:155-160

lithification, B:131

mineral chemistry, B:158-160

modal composition, B:145, 147

petrology, B:158, 160

Site 792, B:169 
tachylite-rich, B:147 temporal trends, B:144-145

sand bed, parallel-laminated, A:330 sand/sandstone, volcaniclastic

geochemistry, B:482

island-arc character, B:471

Izu-Bonin Arc, B:470-474

major-element geochemistry, B:470-474

rare earth elements, B:478

source rocks, B:470-471, 483

tholeiitic affinity, B:479

trace elements, B:475-479

sand/silt, black, Site 790, A:144

sandstone

acid vs. basic components, B:483

alteration, A:334-335; B:106, 109

bed thickness, B:87-90, 95

bedding dips, B:91

Bouma sequences, B:87

color banding, A:346

dacitic composition, B:482

depositional environment, A:246

depositional processes, A:346; B87, 612

diagenesis, B:468

formation microscanner imaging, B:613-617

geochemistry, B:482

graded beds, A:346; B:80-82, 84, 612-614

intraclasts, B:82

magnetic properties, $\mathrm{A}: 84$

mineralogy, B: 105-106, 109

parallel laminations, A:344

petrography, B:126-130

petrology, A:333-334

porosity, A:89

sedimentary structures, B:83

Site 793, B:231

thick beds, B:88-89, 612

trace elements, B:483

X-ray diffraction, A:340, 342

sandstone, calcareous, Site 793, B:234

sandstone, granule

formation microscanner imaging, B:80-82, 86,617

Izu-Bonin forearc, B:612

sandstone, lithic-crystal-vitric, Site 787, A:75

sandstone, muddy

depositional environment, A:244-245, 344

petrography, B:221, 224

Site 792, A:229

Site 793, A:333

sandstone, pebbly

deposition, A:346

formation microscanner imaging, B:80-82, 616

Izu-Bonin forearc, B:612

Site 787, A:73-74

Site 792, A:241

Site 793, A:336

sandstone, pumiceous

parallel laminations, A:336

Site 788, A:111

Site 793, A:343

sandstone, silty

internal structures, A:72-73

Site 787, A:73-74, 76

sandstone, vitric

geochemistry, B:482

graded beds, A:335

logging data, B:657

Miocene, A:407

Site 787, A:72

Site 788, A:109

Site 792, A:229, 235; B:172 sandstone, volcanic, source area, B:482

sandstone, volcaniclastic

diagenetic alteration, B:125-132

geochemistry, B:160

hydrothermal alteration, B:419

Izu-Bonin region, B:155-160

mineral chemistry, B:158-160

petrology, B: 158,160

source areas, B:483

saponite

chemical composition, B:436

magnesium/iron ratio, B:521

Satsuma-Iwojima dacite, sulfur saturation, B:451

Sawa seamounts, B:185

scoria, basalt, seismic reflection profiling, A:132

scour, Site 790, A:154

sea level, changes in, B:94-95

seafloor spreading, Shikoku Basin, B:636, 638

seamounts

cross-arc features, B:564

Izu-Bonin-Mariana Arc, B:639

seawater/rock interaction

basaltic mousse, B:451

Izu-Bonin forearc, B:417, 419, 426, 442-443

sediment gravity flow

calcareous bioclasts in, $\mathrm{B}: 233$

depositional processes, B:87

Izu-Bonin forearc, B:612

mechanism and conditions for, B:91

Oligocene, B:94

pumice deposition by, B:9-10

recurrence intervals, B:89-90

Site 790, B:213

Sumisu Rift, B:228

triggering processes, B:90-91

sediment/igneous transition, physical properties, A:200

sediment sliding, Site 787, B:87

sedimentary clast, Site 793, A:342

sedimentary structures, A:19

Site 788, A:105

Site 791, A:157

Site 793, A:342

sedimentation rate

Brunhes/Matuyama reversal, B:346, 349

Izu-Bonin Arc, B:23, 604

Izu-Bonin forearc, B:604

lithologic correlation, B:497-498

magnetic properties and, B:341

manganese content and, B:498, 502

Oligocene, A:407; B:94

pumice beds, B:7-8

Site 787, A:74-75

Sites 790/791, A:150, 172

Site 792, A:222, 243-244; B:580

Site 793, A:342

Sumisu Rift, B:532, 559, 646

tectonic control, B:569

turbiditic effects on, B:212

volcanic activity and, B:481-482

seismic reflection profiling, A:43-46; B:564, 629

arc margin, B:557, 559

basement, A:6-7

conglomerate-to-nannofossil claystone transition, A:99

correlation, vertical seismic profile, B:578579, 583-584

cross-rift, B:560-561

data acquisition and processing, A:50

forearc, A:51-52

Izu-Bonin arc-trench system, B:630

Izu-Bonin forearc, B:634, 636-637

lithostratigraphic correlation, A:67, 417
Oligocene, B:417, 419

rift basin, B:569

Site 787, A:46-47, 409

Site 788, A:47, 417

Site 789, A:48-49, 417

Site 790, A:44, 46,4849, 120-130, 417

Site 791, A:129-130, 417

Site 792, A:409

Site 793, A:409; B:589

Sumisu Basin N, B:572

Sumisu Rift, A:52; B:557

syn-rift sequence, A:44, 46

Unit I/II boundary, A:129-130

Unit II/III boundary, A:132

VSP correlation, A:314

seismic stratigraphy

Izu-Bonin forearc, B:633, 642

lithostratigraphic correlation, A:103, 129-130, 132, 135, 137, 226, 319; B:576-585

logging correlation, B:579-580, 582-583, 586-587, 590-591

physical property correlation, B:579-580

seismic units 1-3, A:51-52; B:557

seismic units 1-4, A:224-225

seismic units 1-5, B:575, 579-583

Site 787, A:65; B:576-577

Sites 788/789, A:99

Sites 790/791, A:129-130

Site 792, B:577-581

Site 793, A:318; B:581-583

serpentinite seamount

Izu-Bonin forearc, A:6

Mariana forearc, A:6

Shadow Mountain, emplacement, B:563

shear fracture

formation, B:205

Site 793, B:201-202

shear planes, Riedel, B:205

shear strength

ratio to overburden stress, B:546-547

Site 790, A:195, 199

Site 791, A: 199, 200,207

Site 792, A:279, 285

Site 793, A:379, 386, 388

shear zone, Site 787, A:69

Shichiyo seamounts, B:185

Shikinejima Island, white tephra, B:49

Shikoku Basin

backarc spreading, A:5; B:636

magnetic anomalies, B:359, 362

opening, B:95, 364

sandstone-to-mudstone transition, B:95

spreading history, revised, B:636, 638

subduction, B:364

volcanism, B:648

Shikoku-Parece Vela Basin, westward opening, B:362

Shinkurose Ridge, basement highs, B:417, 419, 677

sideromelane, B:140-141

silica. See also magnesium oxide/silica ratio; potassium oxide-silica relationship

ash layers, B:60

backarc vs. forearc sites, B:498-500

basement, A:369

core vs. log measurements, B:593, 597

in glass shards, B:512

logging data, B:526-527, 657

pumice layers, B:514, 516, 525

sinks, B:521-522

Site 787, A:88

Site 793, A:378

tephras, B:71 
vs, potash, $B: 470$

vs. silica, B:471

silica-alkali relationship

ash layers, B:40, 60, 62, 72

pumice beds, B:12

silica phases, dissolution rate, B:525, 528

silica-titanium dioxide relationship, tephras, B:40, 62,73

silicate, framework grains, B:140

silicon

basement, B:434, 437

logging data, A:206

in manganese deposits, B:122-123

sill, diabase. See diabase intrusion

silt, nannofossil-rich clayey

depositional environment, A:244

Site 790, A:144-145

Site 792, A:228

Site 793, A:323

silt, vitric

clayey, A:164; B:213

depositional environment, A:326-327

felsic and mafic components, A:228-229

in pumiceous sand, A:159

Site 790, A:140-141, 144, 157

Site 791, A:147-148, 161

Site 793, A:325

X-ray distraction, A:148, 150

siltstone

depositional processes, B:87, 612

graded beds, B:80

internal structures, A:72-73

$\mathrm{X}$-ray diffraction, A:342

siltstone, clayey vitric, Site 788, A:109

siltstone, lithic-crystal-vitric, Site 787, A:75

siltstone, silty, internal structures, A:72-73

siltstone, vitric

graded beds, A:331-333

Miocene, A:407

Site 787, A:75

Site 788, A:109, 112

Site 792, A:229, 235

Site 793, A:332

Site 53, arc volcanism, B:648

Site 290, foraminifers, B:632

Site 292

Cyclicargolithus floridanus, B:249

Pseudohastigerina micra Zone, B:272

Site 296

arc volcanism, B:639

Cyclicargolithus floridanus, B:249

Globorotalia conoidea, B:276

Globorotalia margaritae, B:273

Globorotalia puncticulata, B:273

location, B:76, 384, 468

Miocene sandstones, B:483

Oligocene deposits, B:94

volcaniclastics, B:632

volcanism, B:636

Zone N8, B:279

Site 393, A:315-403

Site 442

basal sediments, B:636

tephra, B:42, 63, 68

Site 445

diagenetic trends, B:132

Reticulofenestra bisecta, B:247

Site 446, diagenetic trends, B:132

Site 447, sedimentation maximum, B:632

Site 448

Oligocene tholeiitic lava, A:266

volcanism, B:636

Site 449, pyroclastic arc volcanism, B:639
Site 450, pyroclastic arc volcanism, B:639, 648

Site 451, pyroclastic arc volcanism, B:639

Site 453

brown glass, B:145

diagenesis, B:132

fluid geochemistry, A:88

Site 458

bronzite andesite, B:426, 442, 634

Eocene arc tholeiites, A:6

magmatism, B:632

outer arc high, B:630

pelagic sediments, B:632

Site 459

Eocene arc tholeiites, A:6

lavas, B:629

outer arc high, B:630

pelagic sediments, B:632

pyroclastic arc volcanism, B:639

tholeiite-boninite interlayering, B:631

Site 460, tectonic erosion/accretion, B:632

Site 461, tectonic erosion/accretion, B:632

Site 504, sulfur, B:452

Site 709

Gephyrocapsa sp., B:263-265, 267-268

location, B:264

Site 711, Reticulofenestra bisecta, B:247

Site 781, arc-tholeiite intrusion, B:640

Site 782

benthic foraminifers, $\mathrm{B}: 296$

location, B:76, 384, 468, 576

sedimentation rate, $\mathrm{B}: 285$

tephra layers, B:67

Site 783, location, B:576

Site 784

location, B:576

vein structures, B:206

Site 785

location, B:576

pumice deposition, B:9

Site 786

benthic foraminifers, B:296

location, B:76, 384, 468, 576

sedimentation rate, $\mathrm{B}: 285$

volcaniclastic layers, B:68

Site 787, A:63-96. See also density; interstitialwater chemistry; lithostratigraphy; magnetic properties; particular microfossil groups; seismic reflection profiling; seismic stratigraphy; thermal conductivity; $\mathrm{X}$ ray diffraction

arc/forearc development, A:6-9

arc volcanism, B:632

basal sediments, B:633-634

claystone, A:407

deformed beds, B:87

lithologic column, B:50, 157

location, B:76, 156, 196, 321, 384, 450, 468 , $520,543,576$

pelagic/hemipelagic sediments, B:490, 494

pumice deposits, B:9

pyroxene, B:164-166

sedimentary section, B:605, 607

sedimentation, A:408; B:94

seismic stratigraphy, B:576-577

turbidites, A:407

vein structures, $B: 196,198-200$

volcaniclastics, B:155-161, 472, 475

Site 788, A:97-126. See also density; foraminifers; interstitial-water chemistry; lithostratigraphy; physical properties; seismic reflection profiling; seismic stratigraphy; $\mathrm{X}$-ray diffraction

ash layers, B:36-38 glass shards, B:510, 513, 518

location, B:76, 156, 210, 372, 384, 450, 468, $505,520,543,576$

manganese deposits, B:114, 116

olivine, B:162

pelagic/hemipelagic sediments, B:490, 494

pumice deposits, B:8-9, 11

pyroxene, B:164

rift flank uplift, B:559

rifting, A:9

sedimentary succession, A:415; B:506

sedimentation, B:644, 646

trace fossils, B:217-218

volcaniclastic sediment, B:386-387

volcaniclastics, B:155-161, 472, 475

Site 789, A97-126. See also particular microfossil groups; seismic reflection profiling; $\mathrm{X}$ ray diffraction

location, B:156, 372, 450, 505-506, 576

rifting, $\mathrm{A}: 9$

Site 790, A:127-220. See also carbon, organic; density; interstitial-water chemistry; li-

thostratigraphy; magnetic properties; par-

ticular microfossil groups; seismic

reflection profiling; thermal conductivity; $\mathrm{X}$-ray diffraction

amino acids, B:532

arc volcanism, B:646

ash layers, B:28-29, 36-39

basement lava, B:389-391

Brunhes/Matuyama polarity reversal, B:341351

composite stratigraphic section, B:211

Gephyrocapsa sp., B:263-268

glass shards, B:510, 512, 518-522

hydrothermal circulation, B:646

igneous rock, A:415, 418

lithologic column, B:157

location, B:76, 156, 210, 264, 321, 372, 384, $450,468,506,520,532,543,576$

magnetic susceptibility, A:418

olivine, B:162

pelagic/hemipelagic sediments, B:490, 494

pumice, B:4-8

pyroxene, B:164

rift basin, B:559, 562-563

rifting, A:9

sedimentary succession, B:508-509

sedimentation, B:646

sulfur isotopes, B: 450

trace fossils, B:209-215

volcaniclastics, B:155-161, 472, 475, 478

Site 791, A:127-220. See also carbon, organic; density; geochemical logging; lithostratigraphy; magnetic properties; particular microfossils groups; physical properties; seismic reflection profiling; thermal conductivity; X-ray diffraction

amino acids, B:533-534

arc volcanism, B:646

basaltic mousse, B:371-376, 391-392

basement lava, B:389-391

Brunhes/Matuyama polarity reversal, B:341351

composite stratigraphic section, B:211

depositional environment, $\mathrm{B}: 217$

glass shards, B:510, 512, 522-524

hydrothermal circulation, B:646

igneous rock, A:415, 418

lithologic column, B:157

location, B:76, 156, 210, 372, 384, 450, 468, $506,520,532,543,576$

magnetic susceptibility, A:418 
olivine, B:162-163

pelagic/hemipelagic sediments, B:490-491, 494

pumice, B:96

rift basin, B:559, 562-563

rifting, A:9

sedimentary succession, B:508-509

sedimentation, B:646

sulfur isotopes, B:450

tephra, B:36-38

trace fossils, B:215-217

volcaniclastics, B:155-161, 472-479

Site 792, A:221-314. See also carbon, organic; carbonate; density; geochemical logging; geophysical logging; lithostratigraphy; magnetic properties; particular microfossil groups; physical properties; thermal conductivity; X-ray diffraction

arc/forearc development, A:6-9

arc volcanism, B:632

ash layers, B:34-35

basal sediments, B:633-634

basement, A:407; B:419, 421

black tephra, B:63

Brunhes/Matuyama polarity reversal, B:341351

chronostratigraphy, B:679

composite stratigraphic section, B:220

hornblende, B:168

hydrothermal alteration, B:101-107, 419

igneous stratigraphy, B:439-440

lithologic column, B:50, 157, 606

location, B:76, 156, 210, 321, 384, 450, 468, $520,576,593,677$

manganese deposits, B:115-116

marine tephra, B:52-58

mineralogy, B:440-442

Oligocene arc, B:417, 419

Oligocene sedimentary logs, B:78-91

pelagic/hemipelagic sediments, B:491-492, 494-495

pumice deposits, B:9, 11

pyroxene, B:165-167

sedimentary section, B:605, 607

sedimentary structures, B:62, 614, 618-620

sedimentation, A:407-408; B:225-227, 229

seismic stratigraphy, B:577-581

stress field orientation, B:620

sulfur isotopes, B:450-451

tectonic setting, B:171

tephra, B:39, 49, 52-58, 61, 63-66, 457-463

trace fossils, B:218-227

turbidites, A:407; B:608

volcanic stratigraphy, B:405-406

volcaniclastics, B:155-161, 473, 476, 478-479

Site 793, A:315-403. See also carbon, organic; carbonate; geochemical logging; geophysical logging; interstitial-water chemistry; lithostratigraphy; magnetic properties; particular microfossil groups; physical properties; seismic reflection profiling; thermal conductivity; $\mathrm{X}$-ray diffraction

arc/forearc development, A:6-9

arc volcanism, B:632

basement, B:407-417

carbonate bioclasts, B:231-233

chronostratigraphy, B:679

hydrothermal alteration, B:101-107, 436-439

lithologic column, B:157, 232, 606

location, B:76, 156, 196, 321, 450, 468, 520, $543,576,593,678$

manganese deposits, B:115-116 mineralogy, B:432-439

Oligocene sedimentary logs, B:78-91

pelagic/hemipelagic sediments, B:492, 495

petrography, B:406-407

pumice deposits, B:9

pyroxene, B:165-168

rift volcanism, B:405

sedimentary section, B:605, 607

sedimentary structures, B:614-615, 617-620

sedimentation, A:407-408

seismic stratigraphy, B:581-583

stress field orientation, B:620

sulfur isotopes, B:451

tectonic setting, B:171

tephra, B:51, 66

turbidites, A:407; B:608

vein structures, B:196, 198, 200

volcanic rock, B:432

volcanic stratigraphy, B:405-406

volcaniclastics, B:155-161, 473, 476-479

slump unit, magnetic properties, A:84

smectite

Izu-Bonin forearc, B:102

morphology, B:105

secondary production, B:105

Site 792, A:242

Site 793, A:338, 340; B:417

thermodynamic parameters, B:521

sodium

basement, B:434, 437

concentration gradient, B:520

in glass inclusions, B:173, 175

Site 792, A:270

in volcaniclastic sand/sandstones, B:470

sodium chloride, grain density, B:553

sodium oxide

sinks, B:521-522

Site 792, A:266

sodium oxide/magnesium oxide ratio, basement, B: 423

Sofu Gan, submarine volcanoes, B:564

Sofu Gan Tectonic Line, B:634

sonic $\log$

Site 792, A:289

Site 793, A:389

sonic velocity. See velocity

spinel

basement, B:389-390, 399, 435-436, 444

in olivines, B:396

sponges, amino acid composition, B:537

Steens Mountain polarity transition, VGP migra-

stilbite tion, B:346

stability of, B:521

thermodynamic parameters, B:524-525

stress

horizontal stretching field, B:205

orientation, B:619-620, 624

sedimentary structures, B:614, 618-620

strontium

basement, B:417

Site 787, A:88

trace elements, B:421

volcaniclastic sand/sandstones, B:477

strontium isotopes

arc vs. rift, B:398

basement, B:421-422, 426-427

in glass shards, B:461-462, 465

in marine carbonates, B:489, 495

volcanic rocks, B:387, 391

strontium/zirconium ratio, basement, B:416

subduction

Izu-Bonin Arc, A:6
Izu-Bonin vs. Mariana arcs, B:629-630, 647

submarine valley, Izu-Bonin forearc, A:322

subsidence

across transfer zones, B:564

differential, A:155; B:227-228, 559, 646

focusing of, B:566

history, A:8

syndepositional, B:562

sulfate

concentration gradient, B:520-521

gypsum precipitation and, A:378; B:521

Site 787, A:88

Sites 790/791, A:188

Site 792, A:270

sulfide, seawater sulfide origin, B:452

sulfide/total sulfur ratio, Sumisu Rift, B:451-452

sulfur

alteration effects, B:451-452

in igneous rock, B:450-452

logging data, A:206

Mariana Arc, B:449

recycling, B:452

Sites 790/791, A:186-187, 191-193

Site 792, A:268

sulfate/sulfide ratio, B:451

water content and, B:452

sulfur, total

Site 788, A:122

Site 792, A:272-276

Site 793, A:374-378

sulfur isotopes

alteration effects, B:452

arc-to-backarc transitions, B:452

degassing, B:449, 452

fractionation factor, B:451-452

in igneous rock, B:450-452

mid-ocean-ridge basalt vs. ocean-island basalt, B:449

seawater sulfate reduction and, B:452

subduction-related, B:452

water content and, B:452

Sumisu Basin N, seismic reflection profiling, B:572

Sumisu Basin $S$

isopach map, B:562-563, 570

seismic reflection profiling, B:568

Sumisu Caldera S

location, B:557

as sediment source, B:646

Sumisu Jima, volcanoes, B:417, 564

Sumisu Jima Canyon, seismic reflection profiling, A:317

Sumisu Rift

amino acids, B:532-538

arc margin, B:570

arc spreading, B:113

backarc basin basalt, B:642

backarc volcanism, B:394

basement, A:9; B:392-394

bathymetry, A:100, 131, 416; B:5, 287, 506, $558,573,643$

benthic foraminifers, B:288-290

explosively erupted magmas, B:387

fault pattern, B:564

geological setting, B:185, 532

hanging-wall collapse, B:566, 644

horst blocks, A:9

hydrothermal alteration, B:497

hydrothermal circulation, B:642-644

initiation and development, B:3

isostatic rebound, B:566-567, 644

magma, B:394-395

mineral chemistry, B:187-189 
paleobathymetry, B:288-290, 296

paleoenvironment, B:288-290, 296

petrography, B:185-186

prehnite-pumpellyite metamorphism, B:189192

proto-remnant arc, B:559

pumice, B:9-11, 15, 395-398

radiolarians, B:321-330

ridge barrier, $\mathrm{B}: 10-11$

rift-axis volcanism, A:9

rift basin, B:559, 562-563, 569

rift flank uplift, B:642, 644, 646

sediment depocenters, B:557, 563

sediment source, B:569-570

sedimentary structures, B:563-564

sedimentary succession, A:415

sedimentation, B:563, 569-570, 644, 646

seismic reflection profiling, A:52, 57-60, 102, 134; B:560-561

structural evolution, B:557, 564, 566, 568571,642

subsidence, B:227-228, 559, 564, 646

tectonic setting, B:557

tephra, B:69, 72-73

two-stage development, B:566

unconformity age, B:644, 646

vertical motion history, B:646

volcanic rock, B:497

volcaniclastic sand, B:139-140

volcanism, B:388-393, 646

Sumisu Rift basalt (SRB)

geochemistry, B:396

trace elements, B:387, 390-391

Sumisu Rift Rhyolites (SRR), petrology, B:392393

synthetic seismogram, Site 792, A:308

tachylite, B:140-141, 144, 152-153

Takachiho Orogeny, mechanism, B:364

tantalum, in tephra, B:461-462

tectonic erosion

arc margin, B:570

Izu-Bonin-Mariana Arc, B:632

tectonic evolution

anchored slab model, B:362

arc bending, B:362

forearc rotation, B:362-363

full graben stage, B:566, 570, 642

Izu-Bonin forearc, B:362-367

whole plate rotation, B:363-366, 368-369

tectonic rotation

clockwise, B:357, 362

paleomagnetic declination and, B:357

tectonics

extensional, B:583

half-graben, A:9

Izu-Bonin forearc, A:408

onset, B:583

rift-related, B:634

Site 792, A:237

temperature

logging data, A:289, 294, 309, 389

Site 787, A:87

Sites 790/791, A:190

Site 792, A:271, 288

Site 793, A:372

tension gash, Izu-Bonin forearc, B:206

tephra

accumulation rate, $\mathrm{B}: 34$

calc-alkaline, B:62, 459-450, 462

chemical composition, B:34-35, 40, 59-63, 69

colors, B:27, 48-49, 60, 63, 69

derivation, $B: 461$ frequency, B:33, 49, 61-63, 68

geochemistry, B:457-463, 514-515

grain size, B:35-39, 51, 59, 68-69

high-potassium, B:63, 68

Izu-Bonin Arc, B:48-69

low-alkali tholeiite series, B:62

multiple layer, B:27

origin, $\mathrm{B}: 63$

petrography, B:30-32, 64-66

shoshonitic affinity, B:460, 463

simple layer, B:27

source, B:35, 460-463

tantalum, B:461-462

thickness, B:33, 49

tholeiitic affinity, B:459-66

thermal conductivity

hard-rock, A:36

Site 787, A:93, 96

Site 790, A:197-198, 202

Site 791, A:202, 208, 210

Site 792, A:285-287

Site 793, A:388-389

soft-sediment, A:35-36

thermal gravity analysis, Izu-Bonin forearc, B: 115,121

thermodynamics

interstitial waters, B:519-528

mineralogical correlation, B:524-525

thorium, logging data, A:216-220, 289; B:655

thorium/niobium ratio, island-arc tholeiites, B:479

thorium/ytterbium ratio, tephra, B:462

titanium

basement, B:433-434, 437

depletion in tephra, B:461

titanium dioxide

arc-to-bench transition, B:422, 428

basement, A:369; B:445

basement andesite, B:440, 447

core vs. $\log$ measurements, B:593

logging data, B:657

in pelagic/hemipelagic sediments, B:500-501

pumice layers, B:516

tephra layers, B:59

titanium dioxide/magnesium oxide ratio, basement, B:409, 423

titanium dioxide/zirconium ratio, basement, B:416

titanium oxide/magnesium oxide ratio, Site 791, A: 190

titanium oxide/silica ratio, pumice beds, B:12

titanium/vanadium ratio

Site 791, A:190

volcaniclastic sand/sandstones, B:477

titanium/zirconium ratio, vs. neodymium isotopes, B: 428

titanomagnetite, B:347

basement andesite, B:436, 439, 441-442

in diabase intrusives, B:417

todorokite

Izu-Bonin forearc, B:115

Site 788, A:117-120

Tohoku Arc, explosive volcanism, B:63

Tonga, glass inclusions in olivine phenocrysts,

$$
\text { B: } 175
$$

Tonga arc

fossil hydrothermal manganese deposits, B: 113

intraoceanic forearc basement, A:6

Tonga-Fiji region, rhyolitic volcanism, B:514

Torishima, submarine volcanoes, B:564

Torishima Caldera

as pumice source, B:11

as sediment source, B:569

Torishima Rift, backarc basin basalt, B:642
Torishima Volcano

Izu-Bonin diabase association, B:417

as sediment source, B:646

total hydrolyzable amino acids (THAA), in interstitial waters, B:532-535

trace elements

basement andesite, A:370-371

basement basalts, B:398

metamorphic vs. nonmetamorphic rock, B:192

Oligocene transition, B:483

olivine diabase intrusion, A:369

pre-rift arc volcanics, B:388

Site 792, A:266-267; B:419, 426

Site 793, B:409, 417

source relationship, B:426

Sumisu Rift basalts, B:387, 390-391

temporal variation, B:480

volcaniclastic sand/sandstone, B:475-479, 481

trace fossils

anaerobic deposition, B:215, 217, 228

biogenic reworking, B:213, 223, 229

climax, B:226

depositional environment, B:212-220, 223, 225-229

frozen tiers, B:213, 216

killing events, B:220, 226

microscopy, B:221

morphoscopy, B:221

Site 788, B:217-218

Site 790, B:213

Site 791, B:215, 217

Site 792, B:219-223, 225, 227

size, B:222

tectonic evolution, B:228

tiered infaunal community migration, B:225

vertical spreiten migration, B:218

transfer zones

rift oblique, B:570

Sumisu Rift, B:557

uplift/subsidence across, B:564

tuff, crystal-lithic, petrology, A:181, 183-184

tuff, welded, mineralogy, B:105, 109

turbidites

cross lamination, B:612

depositional environment, A:247, 342, 344

depositional rate, A:407

formation microscanner imaging, B:607, 612

incompatible elements, B:388

intraoceanic forearc basins, B:75, 603

Izu-Bonin forearc, B:78, 603

Oligocene, A:407; B:76

physical properties, A:91, 93

ripple-scale cross lamination, B:91-93

Site 787, A:79

Site 792, A:242; B:227

uplift

across transfer zones, B:564

Izu-Bonin-Mariana region, A:8

rift flank, B:566

rifting initiation and, A:418

upward-fining sequence

Izu-Bonin forearc, B:88

source, B:94

tectonic control, A:347-348

uranium, logging data, A:216-220, 289; B:655

vanadium/titanium ratio, volcaniclastic sand/sandstone, B:471

vein structures

anastomosing, Site 793, B:198, 201

backarc settings, B:205

beardlike, B:205 
braided, B:196, 198,206

dip orientation, B:204-205

fish style, B:195, 200, 204

formation, B:205-206

Izu-Bonin forearc, B:195-206

lithologies, B:195

mud, B:196, 198

radiolarian content, B:198, 201

shape and arrangement, B:200-202

sigmoidal, B:198, 203-204, 206

subvertical, B:205-206

tectonic implications, B:205-206

$\mathrm{X}$-ray radiographs, B:197

veinlets

Izu-Bonin forearc, A:408

Site 787, A:69

Site 792, A:246

Site 793, A:333-345

velocity

formation factor and, A:267-268

igneous rock, $\mathrm{A}: 388$

lithologic correlation, A:386, 388

lithostratigraphic correlation, A:201-202

postdepositional processes controlling, B:545

Site 787, A:93, 95

Site 788, A:123, 126

Site 790, A:195, 197, 200

Site 791, A:200-201, 208

Site 792, A:279, 284-285, 312

VSP vs. in situ measurements, B:583-585

velocity/porosity relations, B:545-546

vernadite, Izu-Bonin forearc, B:115

vertical seismic profiles (VSP)

data acquisition, A:294, 301, 395

MCS correlation, A:314

physical properties correlation, B:585

Site 792, A:294, 301, 306, 311, 313; B:582, 585

Site 793, A:389, 395,401

vs. lab and in-situ measurements, A:312 zero offset, B:578-579

virtual geomagnetic pole (VGP), B:342-351

Brunhes/Matuyama reversal, B:345

NRM comparison, B:343

rapid migration, B:342, 345-346

vitrophyric layer, Site 793, A:360

volcanic arc, intraoceanic, formation, A:5

volcanic clasts

in conglomerates, A:333

deposition, A:346

petrology, A:361-362

volcanic front

geochemistry, B:388, 462

Izu-Bonin Arc, B:640

Neogene location, B:386

Pliocene location, B:393

volcanic lithic conglomerate, Site 792, B:172

volcanic lithic fragments

classification, B:140

microlites, B:141-143, 153-154

tachylite grains, B:144

winnowing effects, B:144, 147

volcanic pebbles, geochemistry, A:369

volcanic rock

analytical methods, B:383-385

forearc generational environment, B:422

geochemistry, B:386

Izu-Bonin forearc, B:426

Japanese, B:449

petrography, B:432 stratigraphic units, four types, A:184-185

volcaniclastic-hemipelagic intercalation, Sumisu Rift, B:509, 576

volcaniclastic sediment

age, B:467, 634

arc rhyolite correlation, B:393

physical properties, B:543-549

rift basins, B:557

shear strength to overburden stress ratio, B:546-547

single source, B:515

velocity-porosity relations, B:547

volcaniclastics

depositional processes, B:87

emplacement mechanisms, B:371

formation microscanner imaging, B:78-91

Izu-Bonin forearc, B:75

Quaternary accumulation rate, B:62

sediment failures, B:90

temperature and age, B:528

volcaniclastics, andesitic, geochemistry, B:190

volcaniclastics, unconsolidated, Aoga Shima Canyon, A: 75,77

volcanism, B:514. See also arc volcanism; rift volcanism

acidic, A:346

across transfer zones, B:566

across transform zones, B:644

backarc spreading and, A:8-9

bimodal, B:35, 62-63, 648

Cascadian pulse, B:63

Eocene, B:629-632

in formation of Benin-Mariana forearc, A:6

geochemical history, B:482

history, A:348

intensity and composition, A:318

Izu-Bonin Arc, B:160-161

Izu-Bonin-Mariana Arc, B:648

Izu-Bonin rift, A:9

proto-remnant arc, B:559

structural control, B:555

supra-subduction zone, B:631

tholeiitic/calc-alkaline transition, B:461-462

volcanism, alkalic, syn-rift, B:514

volcanism, andesitic, Sumisu Rift, B:482

volcanism, arc

backarc spreading and, B:47, 62

forearc generation, $\mathrm{B}: 422$

geochemistry, A:8-9

intensity and composition, A:64

Philippine Sea, B:47

volcanism, boninitic, Izu-Bonin forearc, B:426

volcanism, explosive

conditions for, B:371

Izu-Bonin Arc, B:62-63

Site 791, A:159

Site 793, A:346

Sumisu Rift, B:10, 285

volcanism, mafic-felsic, bimodal, A:128

volcanism, rhyolitic, B:512-516

Izu-Bonin Arc, B:49

pre- and syn-rift, B:513-514

provenance, B:515-516

Sumisu Rift, B:646

volcanism, shoshonitic, Mariana Arc, B:463

volcanism, syn-rift

Izu-Bonin forearc, B:647

Izu-Bonin region, A:9

Volcano Arc, strontium isotopes, B:463

volcanoes, paired, B:639 volcanogenic rock, bimodal, A:155, 344

wairakite

alteration temperature, B:107

formation temperature, $\mathrm{B}: 125$

genesis, B:523

Site 792, B:135

stability, B:523

water content

of glass shards, B:512

lithostratigraphic correlation, A:278-279

Site 787, A:91-92

Site 788, A:123, 126

Site 790, A:189

Site 791, A:199

Site 793, A:379

winnowing

Site 787, B:144, 147

Site 788, A:157

wood fragments, B:233

X-ray diffraction

analytical methods, A:30

Izu-Bonin forearc, B:101-104

Site 787, A:74, 78-79

Site 788, A:112-114

Site 790, A:148, 164

Site 791, A:150, 165-166, 184

Site 792, A:240-243, 246-247, 269

Site 793, A:338, 340-342

$\mathrm{X}$-ray fluorescence

analytical methods, A:31-32, 34-35

Site 788, A:121

Site 790, A:121, 188-189

Site 791, A:121, 188-189

Site 792, A:121

Yap Trench, vein structures, B:205

Yellowstone National Park, rhyolite glass, B:510 yttrium

basement, B:419

volcaniclastic sand/sandstones, B:477

yttrium/chromium ratio, Site 793, A:371

yttrium/zirconium ratio, basement, B:416

zeolite. See also cement, zeolite

as calcium sink, B:521

chemical composition, B:438-439

clinoptilolite vs. phillipsite concentration, B:521, 524

elemental composition, B: 125

end-member composition, B:522, 525

Izu-Bonin forearc, B:101, 126-129

secondary production, B:105

Site 792, A:242

smectite covering, B:135

zeolitization, glass and feldspar, B:129

zirconium, volcaniclastic sand/sandstones, B:477

zirconium/barium ratio, B:386

zirconium/yttrium ratio

basement, B:426

Site 792, A:267

Site 793, A:370

volcaniclastic sand/sandstones, B:477, 480

zones, biostratigraphic. See in Taxonomic Index under zones (for letter prefixes) and alphabetically (for generic-specific designations) 


\section{TAXONOMIC INDEX}

abyssorum, Stilostomella, Izu-Bonin Arc, B:307 acostaensis, Globorotalia, Izu-Bonin Arc, B:275 acquilonius, Druppatractus

Izu-Bonin Arc, A:351; B:321, 324

Sumisu Rift, A:167-168; B:322

aculeata, Bulimina

first occurrence, B:298

Izu-Bonin Arc, B:298, 311, 313, 319

paleobathymetry, B:313-314

Sumisu Rift, B:306

acuta, Pleurostomella, Izu-Bonin Arc, B:307

aduncus, Reophax, Izu-Bonin Arc, B:303

affinis, Globobulimina, Sumisu Rift, B:306

Alabamina sp., Izu-Bonin Arc, B:320

altispira, Globoquadrina, Izu-Bonin Arc, B:275276

Ammobaculites sp., Izu-Bonin Arc, B:317

Ammodiscus sp., Izu-Bonin Arc, B:316

Amphicoryna scalaris, Izu-Bonin Arc, B:304

Amphirhopalum ypsilon, Izu-Bonin Arc, B:322

ampliapertura, Globigerina, Izu-Bonin Arc, B:280

angelinum, Axoprunum, Sumisu Rift, A:167-168; $\mathrm{B}: 322$

angusta, Lithocyclia, Izu-Bonin Arc, B:322

annulifera, Stilostomella, Izu-Bonin Arc, B:307

Anomalinoides globulosus, Izu-Bonin Arc, B:308

Anthocyrtidium angulare Zone, Izu-Bonin Arc, A:80; B:322

apicularis, Karrerulina, Izu-Bonin Arc, B:313, 317

arborescens, Dendronina, Izu-Bonin Arc, B:303

asanoi, Reticulofenestra, B:239-240, 243

last occurrence, B:239

Site $790, B: 259$

Site 792, B:261

Asterocyclina sp., Site 793, B:233

Astrononion pusillum, Izu-Bonin Arc, B:311, 320

avita, Didymocyrtis, Site 792, A:254; B:322

Axoprunum angelinum, Sumisu Rift, A:167; B:322

barbata, Bulimina, Sumisu Rift, B:306

barleeanus, Melonis

Izu-Bonin Arc, B:319

Sumisu Rift, B:309

Bathysiphon filiformis, Izu-Bonin Arc, B:318

belemnos, Sphenolithus, Izu-Bonin Arc, A:348; B:247

bengalensis, Osangularia, Izu-Bonin Arc, B:319

berggrenii, Discoaster, Izu-Bonin Arc, A:79; B:249

Biplanispira mirabilis, Site 793, B:87, 231, 233 234

bisecta, Reticulofenestra, Izu-Bonin Arc, A:250; B:247

Bolivina pacifica, Izu-Bonin Arc, B:318

Bolivina pusilla

Izu-Bonin Arc, B:318

Sumisu Rift, B:305

Bolivina robusta, Sumisu Rift, B:305

Bolivinita quadrilatera, Sumisu Rift, B:305

bollii, Discoaster, Site 787, A:79

bollii, Globigerinoides, Izu-Bonin Arc, B:276

boninensis, Globogyroidina, gen. et sp. nov., Sumisu Rift, B:302, 310

boninensis, Nummulites, Hahajima Island, B:233

bradyi, Cibicidoides, Sumisu Rift, B:308

bradyi, Eggerella

Izu-Bonin Arc, B:317
Sumisu Rift, B:303

bradyi, Fissurina, Sumisu Rift, B:304

bradyi, Karreriella, Sumisu Rift, B:303

bradyi, Trifarina, Izu-Bonin Arc, B:319

brevis, Pleurostomella, Izu-Bonin Arc, B:307

Bulimina aculeata

first occurrence, B:298

Izu-Bonin Arc, B:298, 311, 313, 319

paleobathymetry, B:313-314

Sumisu Rift, B:306

Bulimina aculeata Assemblage Zonule

Site 792, A:253; B:292

Sumisu Rift, A:165; B:288

Bulimina aculeata-Uvigerina hispidocostata Assemblage Zonule, Sumisu Rift, A:166; B:288, 290

Bulimina barbata, Sumisu Rift, B:306

Bulimina exilis, Sumisu Rift, B:307

Bulimina rostrata, Izu-Bonin Arc, B:306

Bulimina striata, B:306, 319

Bulimina striata-Bulimina aculeata Assemblage Zonule, Sumisu Rift, A:165-166; B:288

bulloides, Pullenia

Izu-Bonin Arc, B:320

Sumisu Rift, B:310

bulloides, Sphaeroidina

Izu-Bonin Arc, B:311, 319

Sumisu Rift, B:309

Busilla robusta, Izu-Bonin Arc, B:318

calcaris, Discoaster, Site 787, A:79

Calcidiscus leptoporus, Sumisu Rift, B:240, 243, 261

Calcidiscus macintyrei

Izu-Bonin Arc, A:117, 348; B:247

Sumisu Rift, A:160

calculosus, Discoaster, B:247

calida, Globigerina

Izu-Bonin Arc, A:250

Sumisu Rift, B:273

Calocycletta costata Zone, Izu-Bonin Arc, B:324

calyculus, Catinaster, Site 792, A:250; B:247, 249,251,262

cancellata, Cyclammina, Izu-Bonin Arc, B:317

caribbeanica, Gephyrocapsa

classification, B:239

crossbar angles, B:242-243

Sumisu Rift, B:242-244, 246, 259-260

carinata, Cassidulina, Sumisu Rift, B:305

carteri, Helicosphaera, Site 792, B:261

Cassidulina carinata, Sumisu Rift, B:305

Cassidulina sp., Sumisu Rift, B:305

Catapsydrax dissimilis, Izu-Bonin Arc, B:277, 280

Catapsydrax sp., Site 793, A:349

Catapsydrax stainforthi, Izu-Bonin Arc, B:277, 280

Catinaster calyculus, Site 792, A:250; B:247, $249,251,262$

Catinaster coalitus, Site 792, A:250; B:247, 251 , 262

Ceratobulimina pacifica, Izu-Bonin Arc, B:318

cerroazulensis cocoaensis, Globorotalia, IzuBonin Arc, B: 278

Chiloguembelina cubensis, Izu-Bonin Arc, B:277, 280

Chilostomella oolina, Izu-Bonin Arc, B:309, 313, 319
Chilostomella oolina-Uvigerina hispidocostata Assemblage Zonule, Sumisu Rift, A:166; B: 290

Chilostomella ovoidea, Izu-Bonin Arc, B:319

Chondrites

Izu-Bonin Arc, B:218-220, 222-223, 225, 227

Sumisu Rift, B:210, 212-216

Chrysalogonium longicostatum, Izu-Bonin Arc, B:304

Cibicidoides bradyi, Sumisu Rift, B:308

Cibicidoides havanensis, B:308, 319

Cibicidoides mundulus

Izu-Bonin Arc, B:319

Sumisu Rift, B:308

Cibicidoides renzi, Izu-Bonin Arc, B:308

Cibicidoides renzi-Bulimina jarvisi Assemblage Zonule, B:296

Cibicidoides renzi-Cibicidoides wuellerstorfi Assemblage Zonule, Site 792, A:251, 253; B:291

Cibicidoides robertsonianus, Izu-Bonin Arc, B:319

Cibicidoides sp., Izu-Bonin Arc, B:308, 319

Cibicidoides spp.-Epistominella sp. Assemblage Zonule, Site 792, A:253; B:291

Cibicidoides spp.- Rhizammina sp. Assemblage Zonule, Site 793, A:351; B:294, 296

Cibicidoides wuellerstorfi, Izu-Bonin Arc, A:80, 253; B:308, 319

Cibicidoides wuellerstorfi-Pyrgo murrhina Assemblage Zonule, B:296

ciperoensis, Sphenolithus

Izu-Bonin Arc, A:250, 349; B:247, 249, 251

last occurrence, A:349; B:249, 251

Clausicoccus fenestratus, B: 246

Clausicoccus sp., Site 792, B:262

coalitus, Catinaster, Site 792, A:250; B:247, 251, 262

communis, Dentalina, Izu-Bonin Arc, B:304, 318

communis, Martinottiella

Izu-Bonin Arc, B:317

Sumisu Rift, B:303

conglobata, Thalmannammina, Izu-Bonin Arc, B:303

conglobatus, Globigerinoides, Izu-Bonin Arc, B: 272

connecta, Globigerina, Izu-Bonin Arc, B:280

conoidea, Globorotalia, Izu-Bonin Arc, B:272, 275-276

continuosa, Globorotalia, Izu-Bonin Arc, B:277, 280

Coronocyclus sp., Site 792, B:262

crassaformis hessi, Globorotalia, Site 793 . A:349; B:279

Cribrostomoides sp., Izu-Bonin Arc, B:316

crispum, Elphidium, Site 787, A:84

cubensis, Chiloguembelina, Izu-Bonin Arc, B:277, 280

Cyclammina cancellata, Izu-Bonin Arc, B:317

Cyclammina trullissata, Izu-Bonin Arc, B:303, 317

Cyclicargolithus floridanus, B:247, 249, 251

classification, B:239

last occurrence, B:25

Cyrtocapsella japonica, Site 792, A:254; B:323

Cyrtocapsella tetrapera, Site 793, A:351; B:324

Cyrtocapsella tetrapera zone, Izu-Bonin Arc, B:323

Cystammina pauciloculata, Izu-Bonin Arc, B:317 
decoraperta, Globigerina, Izu-Bonin Arc, B:275 deflandrei, Discoaster, Izu-Bonin Arc, B:247, 262 delmontensis, Stichocorys, Site 792, A:254; B:323 Dendronina arborescens, Izu-Bonin Arc, B:303 Dentalina communis, Izu-Bonin Arc, B:304, 318 dentaliniformis, Reophax, Izu-Bonin Arc, B:316 depressa, Globocassidulina, Izu-Bonin Arc, B:318

Diartus hughesi, Izu-Bonin Arc, B:322

Diartus petterssoni Zone, Site 792, A:254; B:323

Didymocyrtis alata zone, Izu-Bonin Arc, B:324

Didymocyrtis antepenultima Zone, Site 787, A: $80 ; \mathrm{B}: 322$

Didymocyrtis avita, Site 792, A:254; B:322

Didymocyrtis laticonus, Izu-Bonin Arc, B:323

Didymocyrtis penultima Zone, Site 792, A:254; B:323

Discammina sp., Izu-Bonin Arc, B:316

Discoaster berggrenii, Izu-Bonin Arc, A:79; B: 249

Discoaster bollii, Site 787, A:79

Discoaster calcaris, Site 787, A:79

Discoaster calculosus, B:247

Discoaster deflandrei, Izu-Bonin Arc, B:247, 262

Discoaster druggii, Izu-Bonin Arc, B:247, 262

Discoaster hamatus, Site 792, A:250; B:247, 249, 251, 262

Discoaster kugleri, Site 792, A:250; B:247, 249, 251, 262

Discoaster loeblichii, Site 787, A:79

Discoaster pansus, Site 792, B:262

Discoaster pentaradiatus, Izu-Bonin Arc, B:644

Discoaster quinqueramus, B:249

Discoaster sp., Site 788, A:117

Discoaster surculus, Site 792, A:249

Discoaster variabilis, Site 792, B:262

disjuncta, Sphaeroidinellopsis, Izu-Bonin Arc, B:279-280

dissimilis, Catapsydrax, Izu-Bonin Arc, B:277, 280

distans, Hormosinella, Sumisu Rift, B:316

distentus, Sphenolithus

Izu-Bonin Arc, A:80, 250, 349; B:247, 249

last recurrence, A:349; B:249

Dorcadospyris alata Zone, Site 792, A:254; B:323

Dorcadospyris ateuchus Zone, Site 787, A:80; B:322

druggii, Discoaster, Izu-Bonin Arc, B:247, 262

Druppatractus acquilonius

Izu-Bonin Arc, A:351; B:321, 324

Sumisu Rift, A:167-168; B:322

druryi, Globigerina, Izu-Bonin Arc, B:280

Eggerella bradyi

Izu-Bonin Arc, B:317

Sumisu Rift, B:303

Eggerella propinqua, Izu-Bonin Arc, B:303

Ehrenbergina pacifica

Izu-Bonin Arc, B:318

Sumisu Rift, B:305

elegans, Hoeglundina

Izu-Bonin Arc, B:318

Sumisu Rift, B:304

elegans, Islandiella, Sumisu Rift, B:305

Elphidium crispum, Site 787, A:84

Emiliania huxleyi

first occurrence, B:7

Izu-Bonin Arc, A:248, 348; B:239

Sumisu Rift, A:161; B:7, 242, 259-260

Epistominella exigua, Izu-Bonin Arc, B:310

ericsonii, Gephyrocapsa, Site 790, B:265

Eulepidina formosa, Izu-Bonin Arc, B:298

Eulepidina sp., Site 793, B:231, 234 exigua, Epistominella, Izu-Bonin Arc, B:310

exilis, Bulimina, Sumisu Rift, B:307

extans, Globorotalia, Izu-Bonin Arc, B:280

Favocassidulina favus, Izu-Bonin Arc, B:305, 318

favus, Fovocassidulina, Izu-Bonin Arc, B:305, 318

fenestratus, Clausicoccus, B:246

filiformis, Bathysiphon, Izu-Bonin Arc, B:318

Fissurina bradyi, Sumisu Rift, B:304

Fissurina formosa, Izu-Bonin Arc, B:318

Fissurina sp.

Izu-Bonin Arc, B:318

Sumisu Rift, B:304

Fissurina subformosa, Sumisu Rift, B:304

floridanus, Cyclicargolithus, B:247, 249, 251

classification, B:239

last occurrence, B:249, 251

fohsi lobata, Globorotalia, Site 793, A:349

formosa, Eulepidina, Izu-Bonin Arc, B:298

formosa, Fissurina, Izu-Bonin Arc, B:318

Gavelinopsis lobatulus, Sumisu Rift, B:309

Gephyrocapsa

classification, B:263-264

morphometric changes, B:263-267

Gephyrocapsa caribbeanica

classification, B:239

crossbar angles, B:242-243

Sumisu Rift, B:242-244, 246, 259-260

Gephyrocapsa ericsonii, Site 790, B:265

Gephyrocapsa oceanica

classification, B:239

Sumisu Rift, B:240, 242-244, 246, 259-260

Gephyrocapsa ornata

morphotypes, B:265

Site 790, B:263-265, 269

Gephyrocapsa parallela, B:239

Gephyrocapsa protohuxleyi

morphometric changes, B:265

Site 790, B:243, 263-265, 269

Gephyrocapsa sp., A:117; B:246

Gephyrocapsa sp., large, B:243, 263-265

Gephyrocapsa sp., small, B:239-243, 259-260, 263-265

abundance patterns, B:243, 264-265, 267-268

Gephyrocapsa spp.

classification, B:237, 239

crossbar angles, B:244

Gephyrocapsa Zone, small, B:246, 265

Globigerapsis sp., Izu-Bonin Arc, B:280

Globigerina ampliapertura, Izu-Bonin Arc, B:280

Globigerina calida

Izu-Bonin Arc, A:250

Sumisu Rift, B:273

Globigerina connecta, Izu-Bonin Arc, B:280

Globigerina decoraperta, Izu-Bonin Arc, B:275

Globigerina druryi, Izu-Bonin Arc, B:280

Globigerina gortanii, Izu-Bonin Arc, B:272

Globigerina multicamerata, Izu-Bonin Arc, B:275

Globigerina nepenthes, Izu-Bonin Arc, A:251; B:273

Globigerina pachyderma acme Zone 1, Site 791, A:163

Globigerina pachyderma acme Zone 2, Site 791, A:163

Globigerina pseudovenezuelana, Izu-Bonin Arc, B: 280

Globigerina ruber, Site 788, A:118

Globigerina senni, Izu-Bonin Arc, B:280

Globigerinita iota

Izu-Bonin Arc, B:274, 279

Sumisu Rift, B:273
Globigerinoides bollii, Izu-Bonin Arc, B:276

Globigerinoides conglobatus, Izu-Bonin Arc, B:272

Globigerinoides immaturus, Izu-Bonin Arc, B:280

Globigerinoides kennetti, Izu-Bonin Arc, B:276

Globigerinoides obliquus extremus, Izu-Bonin Arc, B:272

Globigerinoides ruber

Izu-Bonin Arc, B:274, 279

last occurrence, B:274

Sumisu Rift, B:273

Globigerinoides ruber complex, Site 792, A:250

Globigerinoides tenellus, Izu-Bonin Arc, B:274, 279

globigerinoids, Site 787, A:80

Globobulimina affinis, Sumisu Rift, B:306

Globobulimina pacifica, Izu-Bonin Arc, B:319

Globocassidulina depressa, Izu-Bonin Arc, B:318

Globocassidulina moluccensis

Izu-Bonin Arc, B:318

Sumisu Rift, B:305

Globocassidulina sp., Sumisu Rift, B:305

Globocassidulina subglobosa

Izu-Bonin Arc, B:318

Sumisu Rift, B:305

Globogyroidina boninensis gen. et sp. nov., Sumisu Rift, B:302, 310

Globogyroidina gen. nov., B:300, 302

Globoquadrina altispira, Izu-Bonin Arc, B:275276

Globoquadrina humerosa, Izu-Bonin Arc, B:274

Globoquadrina venezuelana, Izu-Bonin Arc, B:274

Globorotalia acostaensis, Izu-Bonin Arc, B:275

Globorotalia cerroazulensis cocoaensis, IzuBonin Arc, B:278

Globorotalia conoidea, Izu-Bonin Arc, B:272, 275-276

Globorotalia continuosa, Izu-Bonin Arc, B:277, 280

Globorotalia crassaformis hessi, Site 793, A:349; B:279

Globorotalia extans, Izu-Bonin Arc, B:280

Globorotalia fohsi lobata, Site 793, A:349

Globorotalia margaritae, Izu-Bonin Arc, B:272273

Globorotalia mayeri, Izu-Bonin Arc, B:280

Globorotalia menardii, Izu-Bonin Arc, B:276

Globorotalia menardii group, Site 787, A:80

Globorotalia menardii-tumida complex, Site 792 , A: 250

Globorotalia mendacis, Izu-Bonin Arc, B:280 Globorotalia miotumida, Izu-Bonin Arc, B:276

Globorotalia miozea, Izu-Bonin Arc, B:276

Globorotalia opimanana, Izu-Bonin Arc, B:272, 280

Globorotalia opima opima, Izu-Bonin Arc, B:272

Globorotalia peripheroronda, Izu-Bonin Arc, B:276-277, 279-280

Globorotalia plesiotumida, Izu-Bonin Arc, B:272

Globorotalia praescitula, Izu-Bonin Arc, B:279280

Globorotalia pseudomiozea, Izu-Bonin Arc, B:280

Globorotalia puncticulata, Izu-Bonin Arc, B:273

Globorotalia semivera, Izu-Bonin Arc, B:280

Globorotalia siakensis, Izu-Bonin Arc, B:277

Globorotalia tosaensis

Izu-Bonin Arc, A:349; B:274, 279

last occurrence, B:273

Globorotalia truncatulinoides

Izu-Bonin Arc, A:250, 349; B:271-272, 274

Sumisu Rift, B:273 
globosa, Saitoella, gen. et sp. nov., Izu-Bonin Arc, B:300, 309

globulosus, Anomalinoides, Izu-Bonin Arc, B:308 gortanii, Globigerina, Izu-Bonin Arc, B:272 graciliformis, Uvigerina, Izu-Bonin Arc, B:306 gracillima, Stilostomella, Izu-Bonin Arc, B:307 guttifer, Reophax, Izu-Bonin Arc, B:316

Gyroidina sp., Izu-Bonin Arc, B:320

Gyroidinoides neosoldanii, Izu-Bonin Arc, B:310, 320

Gyroidinoides sp., B:310, 320

hamatus, Discoaster, Site 792, A:250; B247, 249, 251, 262

hanzawai, Tosaia, Sumisu Rift, B:306

Haplophragmoides sp., Izu-Bonin Arc, B:316

havanensis, Cibicidoides, B:308, 319

Helicosphaera carteri, Site 792, B:261

Helicosphaera inversa, classification, B:239

Helicosphaera sp., Site 792, B:262

Helicosphaera spp., classification, B:239

Helicosphaera wallichii, Site 792, B:261

Helminthopsis

Izu-Bonin Arc, B:218, 222

Sumisu Rift, B:210, 215

heteromorphus, Sphenolithus, Izu-Bonin Arc, A:348; B:247

hispida, Uvigerina, Sumisu Rift, B:306

hispidocostata, Uvigerina, B:306, 319

Hoeglundina elegans

Izu-Bonin Arc, B:318

Sumisu Rift, B:304

Hormosinella distans, Sumisu Rift, B:316

hosoyaensis, Martinottiella

Izu-Bonin Arc, B:317

Sumisu Rift, B:303

hughesi, Diartus, Izu-Bonin Arc, B:322

humerosa, Globoquadrina, Izu-Bonin Arc, B:274

huxleyi, Emiliania

first occurrence, B:7

Izu-Bonin Arc, A:248, 348; B:239

Sumisu Rift, A:161; B:7, 242, 259-260

Hyperammina sp., Izu-Bonin Arc, B:303

immaturus, Globigerinoides, Izu-Bonin Arc, B:280

inversa, Helicosphaera, classification, B:239

iota, Globigerinita

Izu-Bonin Arc, B:274, 279

Sumisu Rift, B:273

irregularis, Umbellosphaera, Site 790, B:261

Islandiella elegans, Sumisu Rift, B:305

japonica, Cyrtocapsella, Site 792, A:254; B:323

Karreriella bradyi, Sumisu Rift, B:303

Karrerulina apicularis, Izu-Bonin Arc, B:313, 317

kennetti, Globigerinoides, Izu-Bonin Arc, B:276

kugleri, Discoaster, Site 792, A:250; B:247, 249, 251,262

lacunosa, Pseudoemiliania

Izu-Bonin Arc, A:117-118, 248; B:239, 242-243

last occurrence, B:239

Sumisu Rift, A:160-161; B:259

laevis, Lagena, Izu-Bonin Arc, B:318

Lagena laevis, Izu-Bonin Arc, B:318

lamarckiana, Quinqueloculina, Izu-Bonin Arc, $\mathrm{B}: 304,318$

Laticarinina pauperata, Izu-Bonin Arc, B:309

laticonus, Didymocyrtis, Izu-Bonin Arc, B:323
Lenticulina rotulatus, Izu-Bonin Arc, B:304

Lenticulina sp., Izu-Bonin Arc, B:304

lepidula, Stilostomella, Sumisu Rift, B:307

leptoporus, Calcidiscus, Sumisu Rift, B:240, 243, 261

Lithocyclia angusta, Izu-Bonin Arc, B:322

lobatulus, Gavelinopsis, Sumisu Rift, B:309

loeblichii, Discoaster, Site 787, A:79

longicostatum, Chrysalogonium, Izu-Bonin Arc B:304

longiscata, Nodosaria, Izu-Bonin Arc, B:304

lucernula, Pyrgo, Sumisu Rift, B:304

macintyrei, Calcidiscus

Izu-Bonin Arc, A:117, 348; B:247

Sumisu Rift, A:160

margarita, Globorotalia, Izu-Bonin Arc, B:272273

Marginulina sp., Izu-Bonin Arc, B:318

Martinottiella communis

Izu-Bonin Arc, B:317

Sumisu Rift, B:303

Martinottiella hosoyaensis

Izu-Bonin Arc, B:317

Sumisu Rift, B:303

mayeri, Globorotalia, Izu-Bonin Arc, B:280

Melonis barleeanus

Izu-Bonin Arc, B:319

Sumisu Rift, B:309

Melonis pompilioides

Izu-Bonin Arc, B:320

Sumisu Rift, B:309

Melonis sp., Izu-Bonin Arc, B:319

menardii, Globorotalia, Izu-Bonin Arc, B:276

mendacis, Globorotalia, Izu-Bonin Arc, B:280

miotumida, Globorotalia, Izu-Bonin Arc, B:276

miozea, Globorotalia, Izu-Bonin Arc, B:276

mirabilis, Biplanispira, Site 793, B:87, 231, 233 234

moluccensis, Globocassidulina

Izu-Bonin Arc, B:318

Sumisu Rift, B:305

multicamerata, Globigerina, Izu-Bonin Arc, $\mathrm{B}: 275$

multicostata, Rectuvigerina, Izu-Bonin Arc, B:306

mundulus, Cibicidoides

Izu-Bonin Arc, B:319

Sumisu Rift, B:308

murrhina, Pyrgo

Izu-Bonin Arc, B:313, 318

Sumisu Rift, B:304

neosoldanii, Gyroidinoides, Izu-Bonin Arc, $\mathrm{B}: 310,320$

nepenthes, Globigerina, Izu-Bonin Arc, A:251; B:273

nipponensis, Paracassidulina, Izu-Bonin Arc, $\mathrm{B}: 318$

Nodosaria longiscata, Izu-Bonin Arc, B:304

Nodulina sp., Izu-Bonin Arc, B:316

Nummulites boninensis, Hahajima Island, B:233

Nummulites vascus, Izu-Bonin Arc, B:298

Nuttallides truempyi, Site 787, A:80

Nuttallides truempyi-Osangularia mexicana Assemblage Zonule, B:296

obliquus extremus, Globigerinoides, Izu-Bonin Arc, B:272

oceanica, Gephyrocapsa

classification, B:239

Sumisu Rift, B:240, 243-244, 246, 259-260

Oculosiphon sp., Izu-Bonin Arc, B:311, 313, 316 oolina, Chilostomella, Izu-Bonin Arc, B:309, 313, 319

Ophthalmidium pusillum, Izu-Bonin Arc, B:304

opima nana, Globorotalia, Izu-Bonin Arc, B:272, 280

opima opima, Globorotalia, Izu-Bonin Arc, B:272

Oridorsalis umbonatus

Izu-Bonin Arc, B:320

Sumisu Rift, B:310

Oridorsalis umbonatus-Pleurostomella spp. Assemblage Zonule, Site 792, A:251; B:291

Oridorsalis umbonatus-Stilostomella spp. Assemblage Zonule, B:296

ornata, Gephyrocapsa

morphotypes, B:265

Site 790, B:263-265, 269

Osangularia bengalensis, Izu-Bonin Arc, B:319

Osangularia mexicana-Globocassidulina subglobosa Assemblage Zonule, B:296

oshimai, Paracassidulina, Izu-Bonin Arc, B:318

ovoidea, Chilostomella, Izu-Bonin Arc, B:319

pacifica, Bolivina, Izu-Bonin Arc, B:318

pacifica, Ceratobulimina, Izu-Bonin Arc, B:318

pacifica, Ehrenbergina

Izu-Bonin Arc, B:318

Sumisu Rift, B:305

pacifica, Globobulimina, Izu-Bonin Arc, B:319

Palaeophycus

Izu-Bonin Arc, B:218, 222

Sumisu Rift, B:215

pansus, Discoaster, Site 792, B:262

Paracassidulina nipponensis, Izu-Bonin Arc, B:318

Paracassidulina oshimai, Izu-Bonin Arc, B:318

Paracassidulina sagamiensis, Izu-Bonin Arc, $\mathrm{B}: 318$

Parafissurina sp.

Izu-Bonin Arc, B:318

Sumisu Rift, B:304

parallela, Gephyrocapsa, B:239

parvula, Reticulofenestra

morphometric changes, B:265

Site 790, B:264-265, 269

pauciloculata, Cystammina, Izu-Bonin Arc, B:317

pauperata, Laticarinina, Izu-Bonin Arc, B:309

Pellatispira rutteni, Site 793, B:231

pentaradiatus, Discoaster, Izu-Bonin Arc, B:644

pentas, Spongaster, Izu-Bonin Arc, B:322

peregrina, Stichocorys, Site 792, A:254

peripheroronda, Globorotalia, Izu-Bonin Arc, B:276-277, 279-280

Planolites

Izu-Bonin Arc, B:218, 220, 222-225, 227

Sumisu Rift, B:210, 212-213, 215

plesiotumida, Globorotalia, Izu-Bonin Arc, B:272

Pleurostomella acuta, Izu-Bonin Arc, B:307

Pleurostomella brevis, Izu-Bonin Arc, B:307

Pleurostomella praegerontica, Izu-Bonin Arc, B:307

Pleurostomella sp., B:307

Pleurostomella spp., last occurrence, B:298

Pleurostomella spp.- stilostomella spp. Assemblage Zonule, Sumisu Rift, A:165; B:288

Pleurostomella subcylindrica, Izu-Bonin Arc, B:307

pompilioides, Melonis

Izu-Bonin Arc, B:320

Sumisu Rift, B:309

Pontosphaera sp., B:261

praegerontica, Pleurostomella, Izu-Bonin Arc, B:307 
praescitula, Globorotalia, Izu-Bonin Arc, B:279280

primalis, Pulleniatina, Izu-Bonin Arc, B:273

proboscidea, Uvigerina, Izu-Bonin Arc, B:319

propinqua, Eggerella, Izu-Bonin Arc, B:303

protohuxleyi, Gephyrocapsa

morphometric changes, B:265

Site 790, B:243, 263-265, 269

Pseudoemiliania lacunosa

Izu-Bonin Arc, A:117-118, 248; B:239, 242-243

last occurrence, B:239

Sumisu Rift, A:160-161; B:259

Pseudohastigerina micra Zone, Izu-Bonin Arc, $\mathrm{B}: 272$

pseudomiozea, Globorotalia, Izu-Bonin Arc, $\mathrm{B}: 280$

pseudoumbilica, Reticulofenestra

classification, B:239

Site 792, B:262

pseudovenezuelana, Globigerina, Izu-Bonin Arc, B:280

pulchra, Syracosphaera, Site 792, B:261

Pullenia bulloides

Izu-Bonin Arc, B:320

Sumisu Rift, B:310

Pullenia bulloides-Melonis barleeanus Assemblage Zonule, Site 793, A:351; B:296

Pullenia quinqueloba

Izu-Bonin Arc, B:320

Sumisu Rift, B:310

Pullenia riveroi, Sumisu Rift, B:310

Pullenia simplex, Sumisu Rift, B:310

Pullenia sp., Izu-Bonin Arc, B:320

Pulleniatina primalis, Izu-Bonin Arc, B:273

puncticulata, Globorotalia, Izu-Bonin Arc, B:273 pusilla, Bolivina

Izu-Bonin Arc, B:318

Sumisu Rift, B:305

pusillum, Astrononion, Izu-Bonin Arc, B:311, 320

pusillum, Ophthalmidium, Izu-Bonin Arc, B:304

Pyrgo lucernula, Sumisu Rift, B:304

Pyrgo murrhina

Izu-Bonin Arc, B:313, 318

Sumisu Rift, B:304

quadrilatera, Bolivinita, Sumisu Rift, B:305 quinqueloba, Pullenia

Izu-Bonin Arc, B:320

Sumisu Rift, B:310

Quinqueloculina lamarckiana, Izu-Bonin Arc, B:304, 318

Quinqueloculina sp., Sumisu Rift, B:304

quinqueramus, Discoaster, B:249

Rabdamminella sp., Izu-Bonin Arc, B:316 ramosa, Saccorhiza, Izu-Bonin Arc, B:311, 316

Rectuvigerina multicostata, Izu-Bonin Arc, B:306 Recurvoides sp., Izu-Bonin Arc, B:317

renzi, Cibicidoides, Izu-Bonin Arc, B:308

Reophax aduncus, Izu-Bonin Arc, B:303

Reophax dentaliniformis, Izu-Bonin Arc, B:316

Reophax guttifer, Izu-Bonin Arc, B:316

Reophax scorpiurus, Izu-Bonin Arc, B:316

Reophax sp., Izu-Bonin Arc, B:303

Reophax spiculifer, Izu-Bonin Arc, B:316

Reticulofenestra asanoi, B:239-240, 243

last occurrence, B:239

Site $790, \mathrm{~B}: 259$

Site 792, B:261

Reticulofenestra bisecta, Izu-Bonin Arc, A:250; B: 247

Reticulofenestra parvula morphometric changes, B:265

Site 790, B:264-265, 269

Reticulofenestra pseudoumbilica

classification, B:239

Site 792, B:262

Reticulofenestra sp.

Site 792, A:249

Sumisu Rift, A:161-162

Reticulofenestra sp., small, B:262

Reticulofenestra spp., classification, B:239

Reticulofenestra umbilica, B:247

Rhabdammina sp., Izu-Bonin Arc, B:303, 316

Rhabdammina sp. Assemblage Zonule, Site 792, A:251; B:290

Rhabdammina sp.- Stilostomella spp. Assemblage Zonule, Site 792, A:251; B:291

Rhabdammina spp.- Oridorsalis umbonatus Assemblage Zonule, Site 793, A:351; B:294

Rhabdammina spp.- Thalmannammina conglobata Assemblage Zonule, Site 793, B:292

Rhabdammina spp.- Thalmannammina sp. Assemblage Zonule, Site 793, A:349, 351

Rhizammina sp., Izu-Bonin Arc, B:303, 316

Rhizammina sp.-Marginulina sp. Assemblage Zonule, Site 793, A:351

Rhizammina sp.- Rhabdammina sp. Assemblage Zonule, Site 793, B:294

riveroi, Pullenia, Sumisu Rift, B:310

robertsonianus, Cibicidoides, Izu-Bonin Arc, B:319

robusta, Bolivina, Sumisu Rift, B:305

robusta, Busilla, Izu-Bonin Arc, B:318

rostrata, Bulimina, Izu-Bonin Arc, B:306

rotulatus, Lenticulina, Izu-Bonin Arc, B:304

ruber, Globigerina, Site 788, A:118

ruber, Globigerinoides

Izu-Bonin Arc, B:274, 279

last occurence, B:274

Sumisu Rift, B:273

rutteni, Pellatispira, Site 793, B:231

Saccammina sphaerica, Izu-Bonin Arc, B:316

Saccorhiza ramosa, Izu-Bonin Arc, B:311, 316

sagamiensis, Paracassidulina, Izu-Bonin Arc, B:318

Saitoella gen. nov., B:299

Saitoella globosa gen. et sp. nov., Izu-Bonin Arc, $\mathrm{B}: 300,309$

scalaris, Amphicoryna, Izu-Bonin Arc, B:304

schlumbergeri, Sigmoilopsis

Izu-Bonin Arc, B:318

Sumisu Rift, B:304

Scolicia, Site 788, B:218, 228

scorpiurus, Reophax, Izu-Bonin Arc, B:316

seminulina, Sphaeroidinellopsis, Izu-Bonin Arc, $\mathrm{B}: 276$

semivera, Globorotalia, Izu-Bonin Arc, B:280

senni, Globigerina, Izu-Bonin Arc, B:280

senticosa, Uvigerina, Sumisu Rift, B:306

siakensis, Globorotalia, Izu-Bonin Arc, B:277

sibogae, Umbilicosphaera, Sumisu Rift, B:242243, 261

Sigmoilopsis schlumbergeri

Izu-Bonin Arc, B:318

Sumisu Rift, B:304

simplex, Pullenia, Sumisu Rift, B:310

Skolithos, Izu-Bonin Arc, B:218-219, 225, 228

sphaerica, Saccammina, Izu-Bonin Arc, B:316

Sphaeroidina bulloides

Izu-Bonin Arc, B:311, 319

Sumisu Rift, B:309

Sphaeroidina sp., Izu-Bonin Arc, B:309, 319
Sphaeroidinellopsis disjuncta, lzu-Benin Arc, B:279-280

Sphaeroidinellopsis seminulina, Izu-Bonin Arc, B:276

Sphaeroidinellopsis spp., Izu-Bonin Arc, A:80; B: 275

Sphaeroidinellopsis subdehiscens, Izu-Bonin Arc, B:275-276

Sphenolithus belemnos, Izu-Bonin Arc, A:348; B:247

Sphenolithus ciperoensis

Izu-Bonin Arc, A:250, 349; B:247, 249,251

last occurrence, A:349; B:249, 251

Sphenolithus distentus

Izu-Bonin Arc, A:80, 250, 349; B:247, 249

last occurrence, A:349; B:249

Sphenolithus heteromophus, Izu-Bonin Arc, A:348; B:247

spiculifer, Reophax, Izu-Bonin Arc, B:316

Spongaster pentas, Izu-Bonin Arc, B:322

Spongaster pentas pentas Zone, Site 792, A:253

Spongaster pentas Zone, Site 792, A:254; B:322

Spongaster tetras, Izu-Bonin Arc, B:322

stainforthi, Catapsydrox, Izu-Bonin Arc, B:277, 280

Stichocorys delmontensis, Site 792, A:254; B:323

Stichocorys peregrina, Site 792, A:254

Stichocorys wolffii, Izu-Bonin Arc, A:254, 351; B:323-324

Stilostomella abyssorun, Izu-Bonin Arc, B:307

Stilostomella annulifera, Izu-Bonin Arc, B:307

Stilostomella gracillima, Izu-Bonin Arc, B:307

Stilostomella lepidula, Sumisu Rift, B:307

Stilostomella sp.- Hyperammina sp. A Assemblage Zonule, Site 793, B:294

Stilostomella sp.- Nodosaria sp. A Assemblage Zonule, Site 793, A:351

Stilostomella spp.

Izu-Bonin Arc, B:298, 307

last occurrence, B:298

Stilostomella spp.- Cibicidoides wuellerstorfi Assemblage Zonule, Site 792, A:253; B:291

Stilostomella spp.- Oridorsalis umbonatus Assemblage Zonule, Site 792, A:251; B:290

Stilostomella spp.- pleurostomella spp. Assemblage Zonule, Site 792, A:253; B:291

Stilostomella spp.- Pullenia bulloides Assemblage Zonule, Site 793, A:351; B:296

striata, Bulimina, B:306, 319

subcylindrica, Pleurostomella, Izu-Bonin Arc, B:307

subdehiscens, Sphaeroidinellopsis, Izu-Bonin Arc, B:275-276

subformosa, Fissurina, Sumisu Rift, B:304

subglobosa, Globocassidulina

Izu-Bonin Arc, B:318

Sumisu Rift, B:305

surculus, Discoaster, Site 792, A:249

Syracosphaera pulchra, Site 792, B:261

Teichichnus, Site 792, B:225

tenellus, Globigerinoides, Izu-Bonin Arc, B:274, 279

tetrapera, Cyrtocapsella, Site 793, A:351; B:324

tetras, Spongaster, Izu-Bonin Arc, B:322

Textularia sp., Izu-Bonin Arc, B:317

Thalassinoides, Site 792, B:222

Thalmannammina conglobata, Izu-Bonin Arc, B:303

Thalmannammina sp., Izu-Bonin Arc, B:317

Theocorythium trachelium dianae, Sumisu Rift, A:167; B:322

tosaensis, Globorotalia 
Izu-Bonin Arc, A:349; B:274, 279

last occurrence, B:273

Tosaia hanzawai, Sumisu Rift, B:306 trachelium dianae, Theocorythium, Sumisu Rift, A:167; B:322

Trifarina bradyi, Izu-Bonin Arc, B:319

Trochammina sp., Izu-Bonin Arc, B:317

truempyi, Nuttallides, Site 787, A:80

trullissata, Cyclammina, Izu-Bonin Arc, B:303, 317

truncatulinoides, Globorotalia

Izu-Bonin Arc, A:250, 349; B:271-272, 274

Sumisu Rift, B:273

Umbellosphaera irregularis, Site 790, B:261

umbilica, Reticulofenestra, B:247

Umbilicosphaera sibogae, Sumisu Rift, B:242243, 261

umbonatus, Oridorsalis

Izu-Bonin Arc, B:320

Sumisu Rift, B:310

Uvigerina graciliformis, Izu-Bonin Arc, B:306

Uvigerina graciliformis Assemblage Zonule, Site 792, B:291

Uvigerina hispida, Sumisu Rift, B:306

Uvigerina hispidocostata, B:306, 319

Uvigerina proboscidea, Izu-Bonin Arc, B:319

Uvigerina proboscidea Acme Zone, Sites 790/791, A: 165

Uvigerina senticosa, Sumisu Rift, B:306

Uvigerina senticosa Acme Zonule, B:288

Uvigerina sp. Assemblage Zonule, Site 792, A: 253 variabilis, Discoaster, Site 792, B:262

vascus, Nummulites, Izu-Bonin Arc, B:298

venezuelana, Globoquadrina, Izu-Bonin Arc, B:274

wallichii, Helicosphaera, Site 792, B:261

wolffii, Stichocorys, Izu-Bonin Are, A:254, 351; B:323-324

wuellerstorfi, Cibicidoides, Izu-Bonin Arc, A:80, 253; B:308, 319

ypsilon, Amphirhopalum, Izu-Bonin Arc, B:322

zones (with letter prefixes)

CD3, A:80, 253

CD4, A:80, 253

CN1a, Site 792, A:250

CN1c, A:250, 348; B:246

CN2, Site 792, A:250; B:247

CN3, A:348; B:247

CN4, Site 793, A:348; B:247

CN5, Site 792, A:250; B:247

CN6, Site 792, A:250

CN7, Site 792, A:250; B:247

CN8, A:80, 250; B:249

CN9, Site 792, A:250; B:249

CN10, Site 787, A:80

CN11, Site 787, A:80

CN11b, Site 792, A:250

CN12, Site 792, A:249-250

CN12C, Site 788, A:117

CN14, A:161-162, 249, 348

CN15, A:79, 117, 160-162, 248-249, 348
CP17, A:250, 349; B:247

CP18, A:250, 349; B:247

CP19, A:80, 250, 349; B:247

N3, Site 792, A:251; B:277

N4, A:349; B:277

N5, A:251; B:277

N6, A:251; B:280

N7/N8 boundary, B:280

N8, Site 792, A:251

N9, A:251, 349; B:279-280

N10, Site 793, A:349; B:280

N15, B:276

N16, A:80; B:276

N17, A:80, 251; B:272, 276

N18, A:80, 118; B:272-273

N19, A:80, 118; B:272, 275

N20, A:80; B:275

N21, Site 792, A:251; B:274

N22, A:80, 250; B:279

N23, A:162-163, 250, 349

P16, B:273, 277-278

P17, B:277

P18, A:251; B:280

P19, A:251; B:280

P21, A:349; B:272, 277

P22, Site 792, A:251; B:277

Zoophycos

Izu-Bonin Arc, B:218, 222, 225

Sumisu Rift, B:213, 215 